\title{
COHOMOLOGY AND THE CLASSIFICATION OF LIFTINGS
}

BY

\author{
JAMES C. BECKER
}

1. Introduction. In this paper we will be concerned with the problem of homotopy classification of liftings of a map. Suppose that $\beta=(E, B, p)$ is a locally trivial fibre space with fibre $F$. Then, for $f: X \rightarrow B$, there is the set $L(X, f, \beta)$ of homotopy classes of liftings of $f$. Assuming that $L(X, f, \beta)$ is not empty and that $X$ is $(2 n-1)$-coconnected and $F$ is $(n-1)$-connected, we will construct, for $\alpha \in L(X, f, \beta)$, an abelian group structure on $L(X, f, \beta)$ such that $\alpha$ is the zero element. Denote the group by $(L(X, f, \beta), \alpha)$ and the sum of two elements $\gamma_{1}$ and $\gamma_{2}$ by $\gamma_{1}+\alpha \gamma_{2}$.

Given $\alpha_{0}, \alpha_{1} \in L(X, f, \beta)$, the different group structures on $L(X, f, \beta)$ determined by $\alpha_{0}$ and $\alpha_{1}$ are isomorphic in the best possible way. The translation $\left(L(X, f, \beta), \alpha_{1}\right)$ $\rightarrow\left(L(X, f, \beta), \alpha_{0}\right)$ which sends $\gamma$ to $\gamma+_{\alpha_{1}} \alpha_{0}$ is an isomorphism.

A weak form of the classification problem is then to determine the structure of the group $(L(X, f, \beta), \alpha)$.

In $\$ 2$ we define a $B$-cohomology theory where $B$ is a fixed space. These are generalizations of cohomology with local coefficients, and if $B$ is a point, they are generalized cohomology theories as in [9]. For each CW-pair $(X, A)$ and integer $n$, $h^{n}(X, A)$ is a local system of abelian groups over the mapping space $\mathscr{M}(X, B)$. The group assigned to $f \in \mathscr{M}(X, B)$ is denoted by $h^{n}(X, A, f)$.

In $\S 4$ we construct the spectral sequence for a fibre map $\pi: Y \rightarrow X$. This is analogous to Dold's generalization of the Serre spectral sequence [2].

In $\$ \$ 5$ and 6 we define a $B$-spectrum and show how to construct a $B$-cohomology theory from a $B$-spectrum. We then associate to a fibre space $\beta=(E, B, p)$ a $B$-spectrum $\mathscr{S}(\beta)$ in a natural way and define, for $\alpha \in L(X, f, \beta)$, a correspondence

$$
\psi_{\alpha}: L(X, f, \beta) \rightarrow h^{0}(X, f, \mathscr{S}(\beta)),
$$

which, in the stable range, i.e., when $X$ is $(2 n-1)$-coconnected and $F$ is $(n-1)$ connected, is one-one and onto. The group structure on $L(X, f, \beta)$ having $\alpha$ as zero element is obtained by pulling back the group structure on $h^{0}(X, f, \mathscr{S}(\beta))$ via $\psi_{\alpha}$. Then (using the terminology of §7) we show that $L(X, f, \beta)$ has a natural affine group structure.

Suppose that $G$ is a finite group which acts on $X$ and $Y$ and is free on $X$. Let $E(X, Y)$ denote the set of homotopy classes of equivariant maps from $X$ to $Y$. Using a construction of Heller [3] and our previous results, we define an affine

Received by the editors May 22, 1967. 
group structure on $E(X, Y)$, provided $E(X, Y)$ is not empty, $X / G$ is $(2 n-1)$ coconnected and $Y$ is $(n-1)$-connected. Our main result on the structure of $(E(X, Y), \alpha)$ is Theorem (8.13).

In $\$ 9$ and 10 we make use of recent results of Hirsch and Haefliger [4], [5] which reduce the problem of classifying immersions (embeddings) of a closed $n$-dimensional $C^{\infty}$-manifold $M$ in euclidean space $E^{n+k}$ to a problem of classifying equivariant maps. These allow us to define a natural affine group structure on the set $I M^{n+k}(M)\left(E M^{n+k}(M)\right)$ of regular homotopy classes of immersions (isotopy classes of embeddings) of $M$ in $E^{n+k}$ provided the set is not empty and $2 k>n+1$ $(2 k>n+3)$. Using Theorem (8.13) we compute the rank and $p$-primary component, p-odd, of these groups.

In $\$ 11$ we study a question raised by Lashof and Smale [7] as to what classes in $H^{k}(M)$ are realizable as normal classes of an immersion of $M$ into $E^{n+k}$.

2. Cohomology theories. Given a space $X$, let $\bar{X}$ denote the category whose objects are points of $X$ and such that the set of maps $M\left(x_{0}, x_{1}\right), x_{0}, x_{1} \in X$, consists of equivalence classes of paths from $x_{1}$ to $x_{0}$, the equivalence relation being homotopy relative to the end points. A continuous map $f: X_{1} \rightarrow X_{2}$ defines a covariant functor $\bar{f}: \bar{X}_{1} \rightarrow \bar{X}_{2}$ in the obvious way.

Let $\mathscr{A}$ denote the category of abelian groups. A local system of abelian groups over $X$ is a covariant functor $L: \bar{X} \rightarrow \mathscr{A}$. We will denote $L([\sigma])$ by $\sigma_{\#}$ where $[\sigma]$ is an equivalence class of paths.

Suppose that local systems $L_{1}: \bar{X}_{1} \rightarrow \mathscr{A}$ and $L_{2}: \bar{X}_{2} \rightarrow \mathscr{A}$ and a map $f: X_{1} \rightarrow X_{2}$ are given. A homomorphism $\psi$ over $f$ from $L_{1}$ to $L_{2}$ is a natural transformation $\psi: L_{1} \rightarrow L_{2} \bar{f}$.

Let $\mathscr{L}$ denote the category whose objects are pairs $(X, L)$ where $L$ is a local system over $X$ and whose maps are pairs $(f, \psi):\left(X_{1}, L_{1}\right) \rightarrow\left(X_{2}, L_{2}\right)$, where $f: X_{1} \rightarrow X_{2}$ and $\psi$ is a homomorphism over $f$ from $L_{1}$ to $L_{2}$.

Let $\mathscr{P}^{2}$ denote the category of $\mathrm{CW}$-pairs. Fix a space $B$. For any space $X$ let $\mathscr{M}(X, B)$ denote the space with the compact-open topology of maps $f: X \rightarrow B$. For $g: X_{1} \rightarrow X_{2}$ define $\mathscr{M}(g): \mathscr{M}\left(X_{2}, B\right) \rightarrow \mathscr{M}\left(X_{1}, B\right)$ by $\mathscr{M}(g)(f)=f g$.

A $B$-cohomology theory on $\mathscr{P}^{2}$ consists of the following.

(A). For $(X, A) \in \mathscr{P}^{2}, f \in \mathscr{M}(X, B)$ and each integer $n$, an abelian group $h^{n}(X, A, f)$.

(B). For $(X, A) \in \mathscr{P}^{2}$ and $F: I \rightarrow \mathscr{M}(X, B)$, with $F(0)=f_{0}, F(1)=f_{1}$, a homomorphism

$$
F_{\#}: h^{n}\left(X, A, f_{1}\right) \rightarrow h^{n}\left(X, A, f_{0}\right) .
$$

(C). For $g:\left(X_{1}, A_{1}\right) \rightarrow\left(X_{2}, A_{2}\right)$ and $f \in \mathscr{M}\left(X_{2}, B\right)$, a homomorphism

$$
g^{*}: h^{n}\left(X_{2}, A_{2}, f\right) \rightarrow h^{n}\left(X_{1}, A_{1}, f g\right) \text {. }
$$

(D). For $(X, A) \in \mathscr{P}^{2}$ and $f \in \mathscr{M}(X, B)$ a homomorphism

$$
d: h^{n}\left(A,\left.f\right|_{A}\right) \rightarrow h^{n+1}(X, A, f) .
$$


These are to have the following properties.

I. For $(X, A) \in \mathscr{P}^{2}$, the collection $\left\{h^{n}(X, A, f), F_{\#}\right\}, f \in \mathscr{M}(X, B), F \in \mathscr{M}(X, B)^{I}$, is a local system over $\mathscr{M}(X, B)$ which will be denoted by $h^{n}(X, A)$.

II. For $g:\left(X_{1}, A_{1}\right) \rightarrow\left(X_{2}, A_{2}\right)$ the collection $\left\{g^{*}: h^{n}\left(X_{2}, A_{2}, f\right) \rightarrow h^{n}\left(X_{1}, A_{1}, f g\right)\right\}$, $f \in \mathscr{M}\left(X_{2}, B\right)$ is a homomorphism of local systems over $\mathscr{M}(g)$.

Then, for $(X, A) \in \mathscr{P}^{2}$, the collection $\left\{h^{n}\left(A,\left.f\right|_{A}\right),\left.F\right|_{A \#}\right\}, f \in \mathscr{M}(X, B), F \in \mathscr{M}(X, B)^{I}$ is a local system over $\mathscr{M}(X, B)$. Here $\left.F\right|_{A}: I \rightarrow \mathscr{M}(A, B)$ is defined by $\left.F\right|_{A}(t)(a)$ $=F(t)(a), a \in A$.

III. For $(X, A) \in \mathscr{P}^{2}$, the collection $\left\{d: h^{n}\left(A,\left.f\right|_{A}\right) \rightarrow h^{n+1}(X, A, f)\right\}, f \in \mathscr{M}(X, B)$ is a homomorphism of local systems over the identity map $\mathscr{M}(X, B) \rightarrow \mathscr{M}(X, B)$.

IV. The function $\mathscr{H}^{n}: \mathscr{P}^{2} \rightarrow \mathscr{L}$ defined by $\mathscr{H}^{n}(X, A)=\left(\mathscr{M}(X, B), h^{n}(X, A)\right)$ and $\mathscr{H}^{n}(g)=\left(\mathscr{M}(g), g^{*}\right)$ is a contravariant functor.

V. For $g:\left(X_{1}, A_{1}\right) \rightarrow\left(X_{2}, A_{2}\right)$ and $f \in \mathscr{M}\left(X_{2}, B\right)$, the diagram

is commutative.

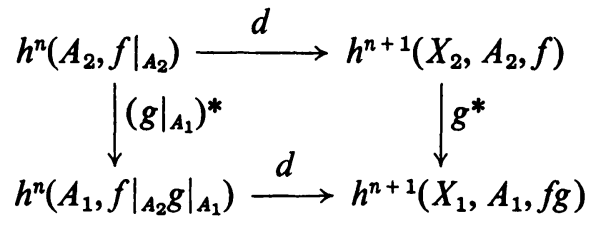

VI. For $G:\left(X_{1}, A_{1}\right) \times I \rightarrow\left(X_{2}, A_{2}\right)$ a homotopy from $g_{0}$ to $g_{1}$, the diagram

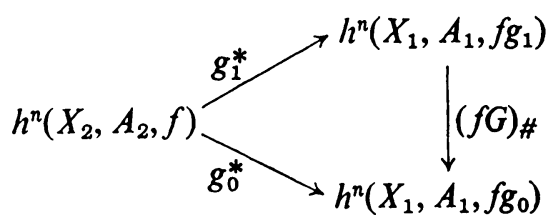

is commutative.

VII. For $(X, A) \in \mathscr{P}^{2}$ and $f \in \mathscr{M}(X, B)$, the sequence

$$
\ldots \stackrel{i^{*}}{\longrightarrow} h^{n}\left(A,\left.f\right|_{A}\right) \stackrel{d}{\longrightarrow} h^{n+1}(X, A, f) \stackrel{j^{*}}{\longrightarrow} h^{n+1}(X, f) \stackrel{i^{*}}{\longrightarrow} \ldots
$$

is exact. Here $j: X \rightarrow(X, A)$ and $i: A \rightarrow X$ are inclusions.

VIII. If $X=A_{1} \cup A_{2}$ and $\left(A_{1}, A_{1} \cap A_{2}\right)$ and $\left(X, A_{2}\right)$ are in $\mathscr{P}^{2}$, then, for $f \in \mathscr{M}(X, B)$,

$$
i^{*}: h^{n}\left(X, A_{2}, f\right) \rightarrow h^{n}\left(A_{1}, A_{1} \cap A_{2},\left.f\right|_{A_{1}}\right)
$$

is an isomorphism. Here $i:\left(A_{1}, A_{1} \cap A_{2}\right) \rightarrow\left(X, A_{2}\right)$ is inclusion.

EXAmple 1. Take $B$ to be a point. Then any generalized cohomology theory (such as in [9]) may be regarded as a $B$-cohomology theory on $\mathscr{P}^{2}$.

For the next example, if $L$ is a local system of abelian groups over $X$, let $H^{n}(X, A ; L)$ denote the $n$th singular cohomology group of $(X, A)$ with coefficients in $L$.

Example 2. Fix a space $B$ and a local system $L$ over $B$. For $(X, A) \in \mathscr{P}^{2}$ and 
$f \in \mathscr{M}(X, B)$, the composition $L \bar{f}: \bar{X} \rightarrow \mathscr{A}$ is a local system over $X$. We then have $H^{n}(X, A ; L \bar{f})$.

For $F: I \rightarrow \mathscr{M}(X, B)$ with $F(0)=f_{0}, F(1)=f_{1}$, and $x \in X$, define $\sigma_{x}: I \rightarrow B$ by $\sigma_{x}(t)=F(x, t), 0 \leqq t \leqq 1$. Then let $F_{\#}=\sigma_{x \#}: L\left(f_{1}(x)\right) \rightarrow L\left(f_{0}(x)\right)$. There results a coefficient homomorphism

$$
F_{\#}: H^{n}\left(X, A, L f_{1}\right) \rightarrow H^{n}\left(X, A, L f_{0}\right) .
$$

With the homomorphism induced by a continuous mapping of pairs and the boundary operator defined in the usual way, it is easy to see that we have a $B$ cohomology theory on $\mathscr{P}^{2}$.

3. Description of $F_{\# \cdot}$ Assume that a cohomology theory over $B$ is given. Axioms IV and VI imply.

(3.1) LEMmA. If $g:\left(X_{1}, A_{1}\right) \rightarrow\left(X_{2}, A_{2}\right)$ is a homotopy equivalence, then for $f \in \mathscr{M}\left(X_{2}, B\right)$,

$$
g^{*}: h^{n}\left(X_{2}, A_{2}, f\right) \rightarrow h^{n}\left(X_{1}, A_{1}, f g\right)
$$

is an isomorphism.

A subspace $A \subset X$ is a weak deformation retract of $X$ if the inclusion $i: A \rightarrow X$ is a homotopy equivalence. By the exact cohomology sequence for $(X, A)$ and the above lemma, we have

(3.2) LemMA. If $A$ is a weak deformation retract of $X$, then for $f \in \mathscr{M}(X, B)$, $h^{n}(X, A, f)=0$.

Now let $f_{0}, f_{1} \in \mathscr{M}(X, B)$ and let $F: X \times I \rightarrow B$ be a homotopy from $f_{0}$ to $f_{1}$. Let $I=\{0,1\}$. We then have a boundary operator

$$
\begin{array}{r}
d_{j}: h^{n}(X \times I \cup A \times I, X \times\{j\} \cup A \times I, F) \rightarrow h^{n+1}(X \times I, X \times I \cup A \times I, F), \\
j=0,1 .
\end{array}
$$

(3.4) LemMa. For $j=0,1, d_{j}$ is an isomorphism.

Proof. By exactness, it is sufficient to show that $h^{n}(X \times I, X \times\{j\} \cup A \times I, F)=0$. This follows from the preceding lemma.

Let $\varepsilon(j)=0$ if $j=1$ and $\varepsilon(j)=1$ if $j=0$. Define $i_{j}:(X, A) \rightarrow(X \times I \cup A \times I$, $X \times\{\varepsilon(j)\} \cup A \times I)$ by $i_{j}(x)=(x, j), j=0,1$. By Axiom VIII,

$$
i_{j}^{*}: h^{n}(X \times I \cup A \times I, X \times\{\varepsilon(j)\} \cup A \times I, F) \rightarrow\left(X, A, f_{j}\right)
$$

is an isomorphism. Hence we have a suspension isomorphism

$$
s_{j}: h^{n+1}(X \times I, X \times I \cup A \times I, F) \rightarrow h^{n}\left(X, A, f_{\varepsilon(j)}\right), \quad j=0,1,
$$

by $s_{j}=i_{\varepsilon(j)}^{*} d_{j}^{-1}$.

Let $\pi: X \times I \rightarrow X$ be the projection. 
(3.7) Lemma. For $f \in \mathscr{M}(X, B)$, the composition

$$
h^{n}(X, A, f) \stackrel{s_{1}^{-1}}{\longrightarrow} h^{n+1}(X \times I, X \times I \cup A \times I, f \pi) \stackrel{s_{0}}{\longrightarrow} h^{n}(X, A, f)
$$

is minus the identity.

The proof is the same as for ordinary cohomology.

The next theorem characterizes $F_{\#}$ in terms of suspension.

(3.8) Theorem. Let $f_{0}, f_{1} \in \mathscr{M}(X, B)$ and let $F: X \times I \rightarrow B$ be a homotopy from $f_{0}$ to $f_{1}$. Commutativity holds in the diagram

$$
h^{n+1}(X \times I, X \times I \cup A \times I, F) \overbrace{s_{1}}^{s_{0}} \overbrace{h^{n}\left(X, A, f_{0}\right)}^{h^{n}\left(X, A, f_{1}\right)}
$$

Proof. Define $M:(X \times I) \times I \rightarrow X \times I$ by $M(x, t)(\lambda)=(x, t \lambda)$. Then $F M_{0}=f_{0} \pi$ and $F M_{1}=F$. We have by Axioms II and III a commutative diagram

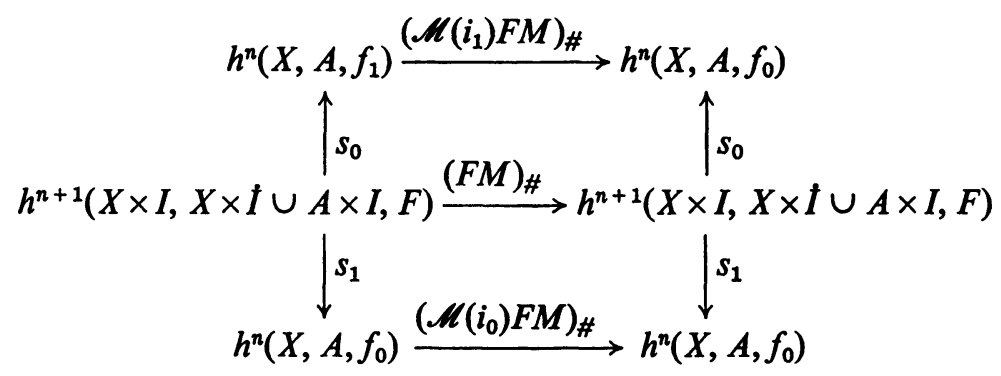

By the previous lemma $s_{1} s_{0}^{-1}$ on the right is minus the identity. Next, we have $\mathscr{M}\left(i_{1}\right) F M=F$ in $\mathscr{M}(X, B)^{I}$ and $\mathscr{M}\left(i_{0}\right) F M$ in $\mathscr{M}(X, B)^{I}$ is the constant path on $f_{0}$. Therefore $\left(\mathscr{M}\left(i_{1}\right) F M\right)_{\#}=F_{\#}$ and $\left(\mathscr{M}\left(i_{0}\right) F M\right)_{\#}$ is the identity. It follows that $s_{1} s_{0}^{-1}$ on the left is $-F_{\# \text {. }}$

Let $\tau_{p}=\left\langle v_{0} \cdots v_{p}\right\rangle$ be an euclidean $p$-simplex and $\tau_{p}$ its boundary. Let $\tau_{p, i}$ $=\left\langle v_{0} \cdots v_{i-1}, v_{i+1} \cdots v_{p}\right\rangle$ and let $J_{i}\left(\tau_{p}\right)$ be the closure of $\tau_{p}-\tau_{p, i}, 0 \leqq i \leqq p$.

For $F \in \mathscr{M}\left(X \times \tau_{p}, B\right)$, define

$$
s_{i}: h^{n}\left(X \times \tau_{p}, X \times \dot{\tau}_{p}, F\right) \rightarrow h^{n-1}\left(X \times \tau_{p, i}, X \times \dot{\tau}_{p, i}, F\right),
$$

$0 \leqq i \leqq p$, to be the composition

$$
\begin{array}{r}
h^{n}\left(X \times \tau_{p}, X \times \dot{\tau}_{p}, F\right) \stackrel{d^{-1}}{\longrightarrow} h^{n-1}\left(X \times t_{p}, X \times J_{i}\left(\tau_{p}\right), F\right) \\
\stackrel{i^{*}}{\longrightarrow} h^{n-1}\left(X \times \tau_{p, i}, X \times \dot{\tau}_{p, i}, F\right) .
\end{array}
$$

Applying $s_{0} p$-times, we obtain

$$
s_{0}^{p}: h^{n}\left(X \times \dot{\tau}_{p}, X \times \dot{\tau}_{p}, F\right) \rightarrow h^{n-p}\left(X \times\left\{v_{p}\right\}, F\right) .
$$


Define $\lambda_{k}: X \rightarrow X \times \tau_{p}$ by $\lambda_{k}(x)=\left(x, v_{k}\right), k=p-1, p$, and let $\pi: X \times \tau_{p} \rightarrow X$ be the projection.

(3.12) LemMa. For $f \in \mathscr{M}(X, B)$, the diagram

$$
\begin{aligned}
& h^{n}\left(X \times \tau_{p}, X \times \dot{\tau}_{p}, f \pi\right) \stackrel{s_{i}}{\longrightarrow} h^{n}\left(X \times \tau_{p, i}, X \times \dot{\tau}_{p, i}, f \pi\right) \\
& \downarrow_{h^{n-p}(X, f) \stackrel{(-1)^{i}}{\longrightarrow} h_{p}^{*} s_{0}^{p-p}(X, f)}^{\downarrow} \lambda_{p}^{*} s_{0}^{p-1}
\end{aligned}
$$

is commutative, where $k=p$ if $0 \leqq i \leqq p$, and $k=p-1$ if $i=p$.

The proof is the same as for ordinary cohomology.

Now for $F \in \mathscr{M}\left(X \times \tau_{p}, B\right)$ consider the diagrams

$$
\begin{aligned}
& h^{n}\left(X \times \tau_{p}, X \times \tau_{p}, F\right) \stackrel{s_{i}}{\longrightarrow} h^{n}\left(X \times \tau_{p, i}, X \times \dot{\tau}_{p, i}, F\right)
\end{aligned}
$$

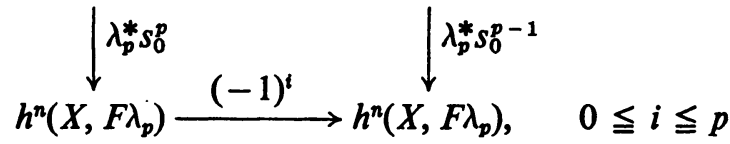

$$
\begin{aligned}
& h^{n}\left(X \times \tau_{p}, X \times \dot{\tau}_{p}, F\right) \stackrel{s_{p}}{\longrightarrow} h^{n}\left(X \times \tau_{p, p}, X \times \dot{\tau}_{p, p}, F\right)
\end{aligned}
$$

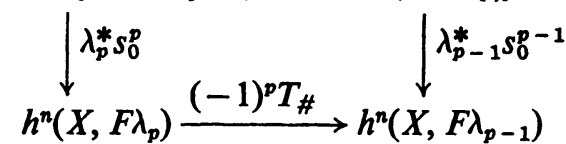

where $T: X \times I \rightarrow B$ is defined by $T(x, t)=F\left(x, t v_{p-1}+(1-t) v_{p}\right), 0 \leqq t \leqq 1$.

(3.15) Lemma. The diagrams (3.13) and (3.14) are commutative.

The proof is similar to the proof of Theorem (3.8). Here we use the preceding lemma, the homotopy $M:\left(X \times \tau_{p}\right) \times I \rightarrow X \times \tau_{p}$ by $M(x, z)(\lambda)=\lambda z+(1-\lambda) v_{p}$, $0 \leqq \lambda \leqq 1$, and a diagram similar to (3.9).

4. The spectral sequence. Assume that a space $B$ and a $B$-cohomology theory on $\mathscr{P}^{2}$ is given. In this section we construct the spectral sequence associated with a fibre map $\pi: Y \rightarrow X$. This is a generalization of the Serre-Dold spectral sequence [2].

We assume that $\pi: Y \rightarrow X$ is locally trivial, $X$ is a polyhedron and for each pair $(K, L)$ of subcomplexes of $X$, we have $\left(\pi^{-1}(K), \pi^{-1}(L)\right) \in \mathscr{P}^{2}$.

Let $f \in \mathscr{M}(X, B)$ be given. Let $F(x)=\pi^{-1}(x), x \in X$. We now describe the way in which the collection of groups $h^{n}(F(x), f \pi), x \in X$, is a local system over $X$. Let $\sigma: I \rightarrow X$ be a path from $x_{0}$ to $x_{1}$. By the covering homotopy property, there is $S(\sigma): F\left(x_{0}\right) \times I \rightarrow X$ such that $S(\sigma)$ covers $\sigma$ and $S(\sigma)_{0}: F\left(x_{0}\right) \rightarrow F\left(x_{0}\right)$ is the identity. We then have $S(\sigma)_{1}: F\left(x_{0}\right) \rightarrow F\left(x_{1}\right)$. 
Next, for $x \in X$, we have $T(x, \sigma): I \rightarrow \mathscr{M}(F(x), B)$ by $T(x, \sigma)(t)(y)=f \sigma(t)$, $0 \leqq t \leqq 1, y \in F(x)$. Note that $T\left(x_{0}, \sigma\right)(1)=f \pi S(\sigma)_{1}$. Let

$$
\sigma_{\#}: h^{n}\left(F\left(x_{1}\right), f \pi\right) \rightarrow h^{n}\left(F\left(x_{0}\right), f \pi\right)
$$

be the composition

$$
h^{n}\left(F\left(x_{1}\right), f \pi\right) \stackrel{S(\sigma)_{1}^{*}}{\longrightarrow} h^{n}\left(F\left(x_{0}\right), f \pi S(\sigma)_{1}\right) \stackrel{T\left(x_{0}, \sigma\right)_{\#}}{\longrightarrow} h^{n}\left(F\left(x_{0}\right), f \pi\right) .
$$

(4.2) LEMMA. The assignment of $h^{n}(F(x), f \pi)$ to $x \in X$ and of $\sigma_{\#}$ to $\sigma \in X^{I}$ is a local system on $X$.

Proof. Let $\sigma$ be a path from $x_{0}$ to $x_{1}$ and $\tau$ a path from $x_{1}$ to $x_{2}$. We will show that $(\sigma \tau)_{\#}=\sigma_{\#} \tau_{\#}$ and leave the other properties to the reader. Consider the diagram

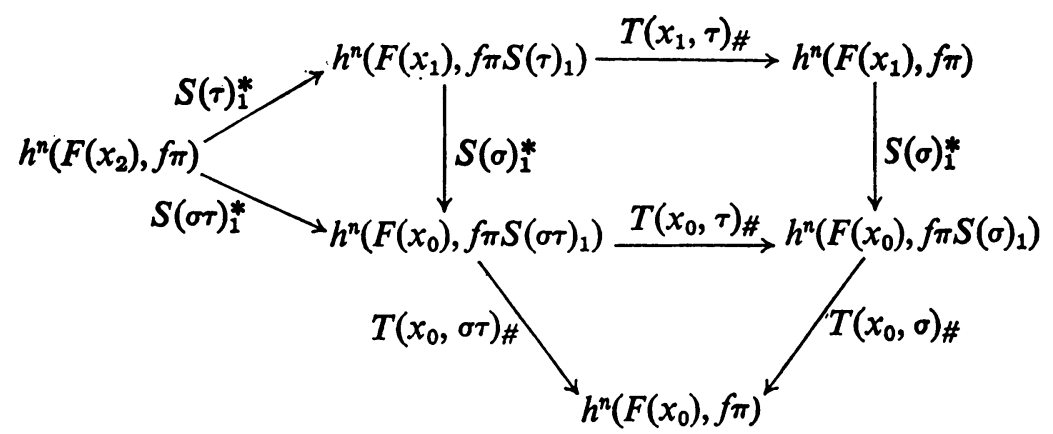

The left hand triangle is commutative, since we may take $S(\sigma \tau)_{1}$ to be the composition $S(\tau)_{1} S(\sigma)_{1}$. The lower triangle is commutative by Axiom I. The square is commutative by Axiom II. Thus $(\sigma \tau)_{\#}=\sigma_{\#} \tau_{\#}$.

We will denote the local system described above by $\left[h^{n}(F)\right]$.

Let $X_{p}$ be the $p$-skeleton of $X$ and let $Y_{p}=\pi^{-1}\left(X_{p}\right)$. We have an exact sequence

$$
\cdots \stackrel{d}{\longrightarrow} h^{n}\left(Y, Y_{p}, f \pi\right) \stackrel{i^{*}}{\longrightarrow} h^{n}\left(Y, Y_{p-1}, f \pi\right) \stackrel{j^{*}}{\longrightarrow} h^{n}\left(Y_{p}, Y_{p-1}, f \pi\right) \stackrel{d}{\longrightarrow} \cdots
$$

Piecing these together leads to an exact couple with

$$
E_{1}^{p, q}=h^{p+q}\left(Y_{p}, Y_{p-1}, f \pi\right), \quad D_{1}^{p, q}=h^{p+q}\left(Y, Y_{p-1}, f \pi\right) .
$$

Fix a total ordering of the vertices of $X$. For $\tau_{p}=\left\langle v_{0} \cdots v_{p}\right\rangle$, define

$$
\tilde{s}_{i}: h^{p+q}\left(\pi^{-1}\left(\tau_{p}\right), \pi^{-1}\left(\dot{\tau}_{p}\right), f \pi\right) \rightarrow h^{p+q-1}\left(\pi^{-1}\left(\tau_{p, i}\right), \pi^{-1}\left(\tau_{p, i}\right), f \pi\right)
$$

to be $i^{*} d^{-1}$, where $d$ is from the cohomology sequence of the triple $\left(\pi^{-1}\left(\tau_{p}\right)\right.$, $\left.\pi^{-1}\left(\tau_{p, i}\right), \pi^{-1}\left(J_{i}\left(\tau_{p}\right)\right)\right)$ and $i:\left(\pi^{-1}\left(\tau_{p, i}\right), \pi^{-1}\left(\tau_{p, i}\right)\right) \rightarrow\left(\pi^{-1}\left(\tau_{p}\right), \pi^{-1}\left(J_{i}\left(\tau_{p}\right)\right)\right)$ is the inclusion.

Applying $\tilde{s}_{0} p$-times leads to an isomorphism

$$
\tilde{s}_{0}^{p}: h^{p+q}\left(\pi^{-1}\left(\tau_{p}\right), \pi^{-1}\left(\dot{\tau}_{p}\right), f \pi \rightarrow h^{q}\left(F\left(v_{p}\right), f \pi\right) .\right.
$$


Consider the diagrams

$$
\begin{aligned}
& h^{p+q}\left(\pi^{-1}\left(\tau_{p}\right), \pi^{-1}\left(\dot{\tau}_{p}\right), f \pi\right) \stackrel{\tilde{S}_{i}}{\longrightarrow} h^{p+q-1}\left(\pi^{-1}\left(\tau_{p, i}\right), \pi^{-1}\left(\tau_{p, i}\right), f \pi\right)
\end{aligned}
$$

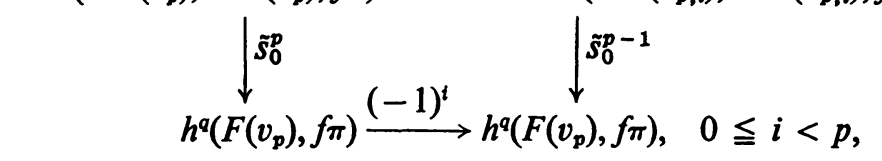

$$
\begin{aligned}
& h^{p+q}\left(\pi^{-1}\left(\tau_{p}\right), \pi^{-1}\left(\dot{\tau}_{p}\right), f \pi\right) \stackrel{\tilde{S}_{p}}{\longrightarrow} h^{p+q-1}\left(\pi^{-1}\left(\tau_{p, p}\right), \pi^{-1}\left(\dot{\tau}_{p, p}\right), f \pi\right) \\
& \underset{h^{q}\left(F\left(v_{p}\right), f \pi\right) \stackrel{(-1)^{p} \sigma_{\#}}{\longrightarrow} h^{q}\left(F\left(v_{p-1}^{p}\right), f \pi\right)}{\downarrow}
\end{aligned}
$$

where $\sigma: I \rightarrow X$ is defined by $\sigma(t)=t v_{p}+(1-t) v_{p-1}, 0 \leqq t \leqq 1$.

(4.8) LemMA. The diagrams (4.6) and (4.7) are commutative.

Proof. We will show that (4.7) is commutative. Choose $S: F\left(v_{p-1}\right) \times \tau_{p} \rightarrow$ $\pi^{-1}\left(\tau_{p}\right)$ to cover the inclusion $\tau_{p} \subset X$ and such that $S \lambda_{p-1}: F\left(v_{p-1}\right) \rightarrow F\left(v_{p-1}\right)$ is the identity. We have a commutative diagram

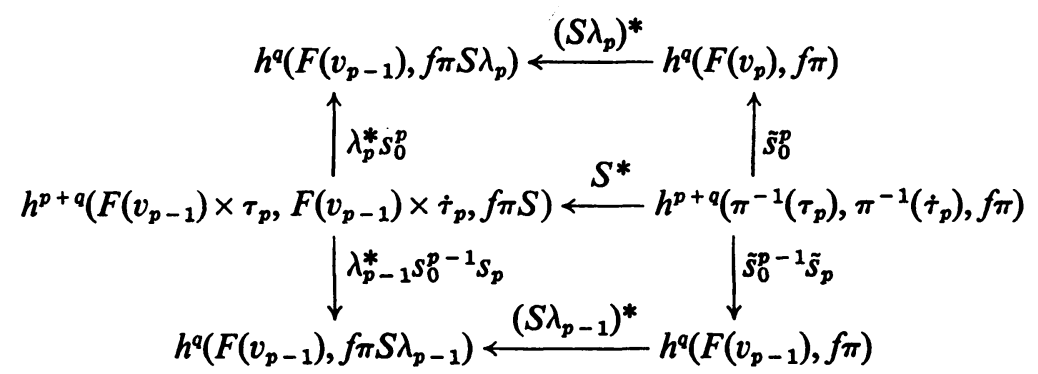

Now use this, the commutativity of (3.14) and the fact that $S \lambda_{p-1}$ is the identity to deduce that

$$
\tilde{s}_{0}^{p-1} \tilde{s}_{p}=(-1)^{p} T_{\#}\left(S \lambda_{p}\right)^{*} \tilde{s}_{0}^{p}
$$

Next, note that $T=T\left(v_{p-1}, \sigma\right)$. Therefore $T_{\#}\left(S \lambda_{p}\right)^{*}=\sigma_{\#}$. The proof that (4.6) is commutative is similar.

For $\tau_{p} \subset X$, let $i\left(\tau_{p}\right): \pi^{-1}\left(\tau_{p}\right) \rightarrow Y_{p}$ be the inclusion. We have

$$
i\left(\tau_{p}\right)^{*}: h^{p+q}\left(Y_{p}, Y_{p-1}, f \pi\right) \rightarrow h^{p+q}\left(\pi^{-1}\left(\tau_{p}\right), \pi^{-1}\left(\dot{\tau}_{p}\right), f \pi\right) .
$$

Let $C^{*}\left(X ;\left[h^{q}(F)\right]\right)$ denote the simplicial cochain complex of $X$ with coefficients in $\left[h^{q}(F)\right]$. Define

$$
\psi: h^{p+q}\left(Y_{p}, Y_{p-1}, f \pi\right) \rightarrow C^{p}\left(X ;\left[h^{q}(F)\right]\right)
$$

by $\psi(u)\left(\tau_{p}\right)=\tilde{s}_{0}^{p} i\left(\tau_{p}\right)^{*}(u), u \in h^{p+q}\left(Y_{p}, Y_{p-1}, f \pi\right)$. Then $\psi$ is an isomorphism and, by Lemma (4.8), commutes with the boundary operator. Therefore we have an identification

$$
\psi: E_{2}^{p, q} \rightarrow H^{p}\left(X ;\left[h^{q}(F)\right]\right)
$$


We have a filtration

$$
h^{n}(Y, f \pi)=J^{0, n} \supset \ldots \supset J^{p, n-p} \supset \cdots,
$$

where

$$
J^{p, n-p}=\operatorname{Image}\left(h^{n}\left(Y, Y_{p-1}, f \pi\right) \rightarrow h^{n}(Y, f \pi)\right) .
$$

As usual, let $E_{\infty}^{p, n-p}=J^{p, n-p} / J^{p+1, n-p-1}$. We will discuss now the convergence of $\left\{E_{r}, d_{r}\right\}$ to $E_{\infty}$.

Definition. A pair $(X, A)$ is $k$-coconnected if for every local system $L$ of abelian groups over $X$, we have $H^{q}(X, A ; L)=0, q \geqq k$.

(4.13) LEMMA. If $Y$ is $k$-coconnected and $F(x), x \in X$, is l-coconnected, then $D_{r}^{p, q}=0, r>\max (k+2-p, l+1)$.

Proof. By inspecting the singular cohomology spectral sequence of $\pi: Y_{s} \rightarrow Y_{s}$, we see that $Y_{s}$ is $(s+l)$-coconnected. Therefore $\left(Y, Y_{p-1}\right)$ is s-coconnected if $s>\max (k, p-1+l)$. Now take $r>\max (k+2-p, l+1)$. Then $p+r-2>$ $\max (k, p-1+l)$ so that by obstruction theory, there is $M: Y \times I \rightarrow Y$ such that $M_{0}$ is the identity, $M_{1}(Y) \subset Y_{p+r-2}$, and $M_{t}$ restricted to $Y_{p-1}$ is the identity, $0 \leqq t \leqq 1$. We have a commutative diagram

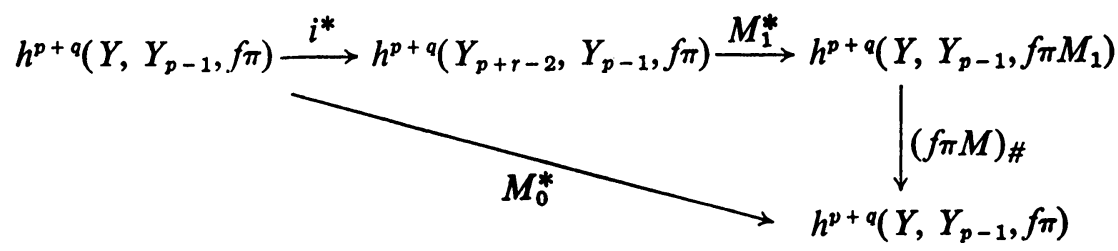

which implies that $i^{*}$ is injective. By exactness,

$$
D_{r}^{p, q}=\operatorname{Image}\left(h^{p+q}\left(Y, Y_{p+r-2}, f \pi\right) \rightarrow h^{p+q}\left(Y, Y_{p-1}, f \pi\right)\right)=0 .
$$

(4.14) Theorem. Suppose that either (a) $X$ is finitely coconnected or (b) $Y$ and $F(x), x \in X$, are finitely coconnected. Then

(1) For each pair $(p, q)$, there is an integer $r(p, q)$ such that $E_{r(p, q)}^{p, q} \simeq E_{\infty}^{p, q}$.

(2) The filtration (4.12) is finite.

This follows by a standard spectral sequence argument. For case (b), the preceding lemma is needed.

5. Liftings. Suppose that we have a pair $(\beta, \Delta)$ where $\beta=(E, B, p)$ is a Serre fibre space and $\Delta: B \rightarrow E$ is a cross-section $\left(p \Delta=\right.$ identity). For $(X, A) \in \mathscr{P}^{2}$, let

$$
\mathscr{L}(X, A, \beta, \Delta)=\left\{g: X \rightarrow E \mid g_{\mid A}=\Delta p g_{\mid A}\right\}
$$

and define

$$
\omega: \mathscr{L}(X, A, \beta, \Delta) \rightarrow \mathscr{M}(X, B)
$$

by $\omega(g)=p g$. 
(5.3) LEMMA. The map $\omega$ is a Serre fibre map.

This follows easily from the exponential law and the fact that $p$ is a Serre fibre map.

Note that $\omega$ has a cross-section

$$
\delta: \mathscr{M}(X, B) \rightarrow \mathscr{L}(X, A, \beta, \Delta)
$$

defined by $\delta(f)=\Delta f$.

The fibre above $f \in \mathscr{M}(X, B)$ will be denoted by $\mathscr{L}(X, A, f, \beta, \Delta)$. When we speak of the homotopy groups of $\mathscr{L}(X, A, f, \beta, \Delta)$, it will be understood that the basepoint is $\Delta f$.

Let $F: I \rightarrow \mathscr{M}(X, B)$ be a path from $f_{0}$ to $f_{1}$. Then $\delta F$ is a path in $\mathscr{L}(X, A, \beta, \Delta)$ from $\Delta f_{0}$ to $\Delta f_{1}$. We have

$$
(\delta F)_{\#}: \pi_{n}\left(\mathscr{L}(X, A, \beta, \Delta) ; \Delta f_{1}\right) \rightarrow \pi_{n}\left(\mathscr{L}(X, A, \beta, \Delta) ; \Delta f_{0}\right) .
$$

Now define

$$
F_{\#}: \pi_{n}\left(\mathscr{L}\left(X, A, f_{1}, \beta, \Delta\right)\right) \rightarrow \pi_{n}\left(\mathscr{L}\left(X, A, f_{0}, \beta, \Delta\right)\right)
$$

so that the diagram

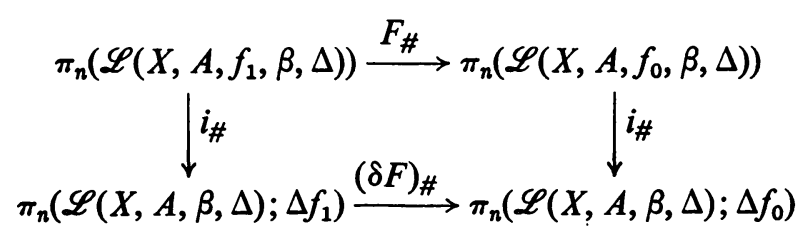

is commutative. (This is possible because of the cross-section $\delta$.) Then, as in [1], we have

(5.7) LEMMA. The correspondence $f \rightarrow \pi_{n}(\mathscr{L}(X, A, f, \beta, \Delta))$ and $F \rightarrow F_{\#}$ is a local system on $\mathscr{M}(X, B)$.

We consider the effect of a change of variable. For $g:\left(X_{1}, A_{1}\right) \rightarrow\left(X_{2}, A_{2}\right)$; we have a commutative diagram

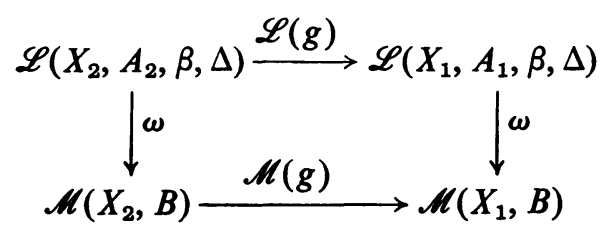

where $\mathscr{L}(g)(h)=h g$. Therefore the collection

$$
\mathscr{L}(g)_{\#}: \pi_{n}\left(\mathscr{L}\left(X_{2}, A_{2}, f, \beta, \Delta\right)\right) \rightarrow \pi_{n}\left(\mathscr{L}\left(X_{1}, A_{1}, f g, \beta, \Delta\right)\right),
$$

$f \in \mathscr{M}\left(X_{2}, B\right)$, is a homomorphism of local systems.

Now let $\left(\beta_{1}, \Delta_{1}\right)$ and $\left(\beta_{2}, \Delta_{2}\right)$ be given where $\beta_{i}=\left(E_{i}, B, p_{i}\right), i=1,2$. By a map

$$
(k, K):\left(\beta_{1}, \Delta_{1}\right) \rightarrow\left(\beta_{2}, \Delta_{2}\right)
$$


we mean $k: E_{1} \rightarrow E_{2}$ such that $p_{2} k=p_{1}$ and $K: B \times I \rightarrow E_{2}$ such that $K_{0}=\Delta_{2}$, $K_{1}=k \Delta_{1}$, and $p_{2} K_{t}=$ identity, $0 \leqq t \leqq 1$. Thus, up to the homotopy $K, k$ is crosssection preserving. We have a commutative diagram

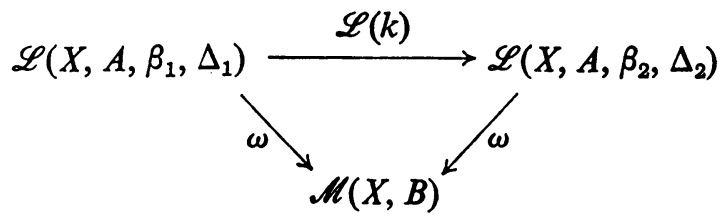

where $\mathscr{L}(k)(h)=k h$. Note that for $f \in \mathscr{M}(X, B)$, the composition $I \stackrel{K}{\rightarrow} \mathscr{M}\left(B, E_{2}\right)$ $\stackrel{\mathscr{M}(f)}{\longrightarrow} \mathscr{M}\left(X, E_{2}\right)$ is a path in $\mathscr{L}\left(X, A, \beta_{2}, \Delta_{2}\right)$ from $\Delta_{2} f$ to $k \Delta_{1} f$. Define

$$
(k, K)_{\#}: \pi_{n}\left(\mathscr{L}\left(X, A, f, \beta_{1}, \Delta_{1}\right)\right) \rightarrow \pi_{n}\left(\mathscr{L}\left(X, A, f, \beta_{2}, \Delta_{2}\right)\right),
$$

$f \in \mathscr{M}(X, B)$, to be the composition

$$
\begin{aligned}
& \pi_{n}\left(\mathscr{L}\left(X, A, f, \beta_{1}, \Delta_{1}\right)\right) \stackrel{\mathscr{L}(k)_{\#}}{\longrightarrow} \pi_{n}\left(\mathscr{L}\left(X, A, f, \beta_{2}, \Delta_{2}\right) ; k \Delta_{1} f\right) \\
& \downarrow(\mathscr{M}(f) K)_{\#} \\
& \pi_{n}\left(\mathscr{L}\left(X, A, f, \beta_{2}, \Delta_{2}\right)\right) \text {. }
\end{aligned}
$$

It is easy to show that $(k, K)_{\#}$ is a homomorphism of local systems.

6. $B$-spectra. Given $(\beta, \Delta)$ as in the previous section, let

(6.1) $\Omega(E ; \Delta)=\left\{\sigma: I \rightarrow E \mid \sigma(I) \subset p^{-1}(b)\right.$, some $b \in B$, and $\left.\sigma(0)=\sigma(1)=\Delta(b)\right\}$ and define

$$
\Omega(p): \Omega(E ; \Delta) \rightarrow B
$$

by $\Omega(p)(\sigma)=b$, where $\sigma(I) \subset p^{-1}(b)$. Using the exponential law we see that $\Omega(\beta ; \Delta)$ $=(\Omega(E ; \Delta), B, \Omega(p))$ is a Serre fibre space. Define a cross-section

$$
\Omega(\Delta): B \rightarrow \Omega(E ; \Delta)
$$

by $\Omega(\Delta)(b)(t)=\Delta(b), 0 \leqq t \leqq 1$.

The pair $(\Omega(\beta ; \Delta), \Omega(\Delta))$ will be called the loop space of $(\beta, \Delta)$.

For $(X, A) \in \mathscr{P}^{2}$ and $f \in \mathscr{M}(X, B)$, the exponential law gives an identification

$$
\mathscr{L}(X, A, f, \Omega(\beta ; \Delta), \Omega(\Delta)) \rightarrow \Omega(\mathscr{L}(X, A, f, \beta, \Delta)) .
$$

This in turn leads to an identification

$$
\pi_{r}\left(\mathscr{L}(X, A, f, \Omega(\beta ; \Delta), \Omega(\Delta)) \rightarrow \pi_{r+1}(\mathscr{L}(X, A, f, \beta, \Delta)) .\right.
$$

A $B$-spectrum $\mathscr{S}$ is a sequence of pairs $\left(\beta_{m}, \Delta_{m}\right),-\infty<m<+\infty$, where $\beta_{m}$ $=\left(E_{m}, B, p_{m}\right)$ is a Serre fibre space and $\Delta_{m}: B \rightarrow E_{m}$ is a cross-section, together with maps

$$
\left(k_{m}, K_{m}\right):\left(\beta_{m}, \Delta_{m}\right) \rightarrow\left(\Omega\left(\beta_{m+1} ; \Delta_{m+1}\right), \Omega\left(\Delta_{m+1}\right)\right) .
$$


Given a $B$-spectrum $\mathscr{S}$, we have for $(X, A) \in \mathscr{P}^{2}$ and $f \in \mathscr{M}(X, B)$, a homomorphism

$$
\left(k_{m}, K_{m}\right)_{\#}: \pi_{n}\left(\mathscr{L}\left(X, A, f, \beta_{m}, \Delta_{m}\right)\right) \rightarrow \pi_{n+1}\left(\mathscr{L}\left(X, A, f, \beta_{m+1}, \Delta_{m+1}\right)\right)
$$

(using the identification (6.5)). Now, for each integer $n$, let

$$
h^{n}(X, A, f ; \mathscr{S})=\operatorname{dir} \lim _{m} \pi_{-n+m}\left(\mathscr{L}\left(X, A, f, \beta_{m+1}, \Delta_{m+1}\right)\right) .
$$

Given $F: I \rightarrow \mathscr{M}(X, B)$ from $f_{0}$ to $f_{1}$, the homomorphisms

$$
F_{\#}: \pi_{n}\left(\mathscr{L}\left(X, A, f_{1}, \beta_{m}, \Delta_{m}\right)\right) \rightarrow \pi_{n}\left(\mathscr{L}\left(X, A, f_{0}, \beta_{m}, \Delta_{m}\right)\right)
$$

commute with those in (6.7). Let

$$
F_{\#}: h^{n}\left(X, A, f_{1} ; \mathscr{S}\right) \rightarrow h^{n}\left(X, A, f_{0} ; \mathscr{S}\right)
$$

be obtained from these by passing to the direct limit.

Given $g:\left(X_{1}, A_{1}\right) \rightarrow\left(X_{2}, A_{2}\right)$ and $f \in \mathscr{M}\left(X_{2}, B\right)$, the homomorphisms

$$
\mathscr{L}(g)_{\#}: \pi_{n}\left(\mathscr{L}\left(X_{2}, A_{2}, f, \beta_{m}, \Delta_{m}\right)\right) \rightarrow \pi_{n}\left(\mathscr{L}\left(X_{1}, A_{1}, f g, \beta_{m}, \Delta_{m}\right)\right)
$$

commute with those in (6.7). Define

$$
g^{*}: h^{n}\left(X_{2}, A_{2}, f ; \mathscr{S}\right) \rightarrow h^{n}\left(X_{1}, A_{1}, f g ; \mathscr{S}\right)
$$

to be the direct limit of the $\mathscr{L}(\mathrm{g})_{\# \text {. }}$

For $(X, A) \in \mathscr{P}^{2}$ let $i: A \rightarrow X$ and $j: X \rightarrow(X, A)$ be the inclusions. Then for $f \in \mathscr{M}(X, B)$ we have

$$
\mathscr{L}\left(X, A, f, \beta_{m}, \Delta_{m}\right) \stackrel{\mathscr{L}(j)}{\longrightarrow} \mathscr{L}\left(X, f, \beta_{m}, \Delta_{m}\right) \stackrel{\mathscr{L}(i)}{\longrightarrow} \mathscr{L}\left(A,\left.f\right|_{A}, \beta_{m}, \Delta_{m}\right)
$$

Using the exponential law we see that $\mathscr{L}(i)$ is a fibre map. Further, $\mathscr{L}(j)$ is an identification with the fibre $\mathscr{L}(i)^{-1}\left(\left.\Delta_{m} f\right|_{A}\right)$. Therefore, we have an exact sequence

$$
\begin{aligned}
\ldots \stackrel{\mathscr{L}(i)_{\#}}{\longrightarrow} \pi_{n}\left(\mathscr{L}\left(A,\left.f\right|_{A}, \beta_{m}, \Delta_{m}\right)\right) \stackrel{\delta_{\#}}{\longrightarrow} \pi_{n-1}\left(\mathscr{L}\left(X, A, f, \beta_{m}, \Delta_{m}\right)\right) \\
\stackrel{\mathscr{L}(j)_{\#}}{\longrightarrow} \pi_{n-1}\left(\mathscr{L}\left(X, f, \beta_{m}, \Delta_{m}\right)\right) \stackrel{\mathscr{L}(i)_{\#}}{\longrightarrow} \ldots
\end{aligned}
$$

and the homomorphisms $\delta_{\#}$ commute with those in (6.7). Therefore we may define

$$
d: h^{n}\left(A,\left.f\right|_{A} ; \mathscr{S}\right) \rightarrow h^{n+1}(X, A, f ; \mathscr{S})
$$

to be the direct limit of the $\delta_{\#}$.

(6.16) THEOREM. With $h^{n}$, \#, *, and d as defined in (6.8), (6.10), (6.12) and (6.15) we have a B-cohomology theory on $\mathscr{P}^{2}$.

Proof. Using the results of $\S 5$, Axioms I through VI are easily checked. Axiom VII follows from the exactness of (6.14) and the fact that exactness is preserved 
under direct limit. For Axiom VIII, note that if $i:\left(A_{1}, A_{1} \cap A_{2}\right) \rightarrow\left(X, A_{2}\right)$ is the inclusion, then for $f \in \mathscr{M}(X, B)$,

$$
\mathscr{L}(i): \mathscr{L}\left(X, A_{2}, f, \beta_{m}, \Delta_{m}\right) \rightarrow \mathscr{L}\left(A_{1}, A_{1} \cap A_{2},\left.f\right|_{A}, \beta_{m}, \Delta_{m}\right)
$$

is a homeomorphism.

7. The group structure. The suspension $S(F)$ of a space $F$ will be the quotient obtained from $F \times I$ by the identification

$$
(x, t) \sim\left(x^{\prime}, t\right) \text { if and only if } x=x^{\prime} \text { or } t=0 \text { or } t=1 \text {. }
$$

However, we will use a weaker topology than the usual one. Let $\omega: F \times I \rightarrow S(F)$ denote the projection. A basis for the topology on $S(F)$ is to consist of sets of the form $\omega\left(U \times\left(t_{1}, t_{2}\right)\right), U$ open in $F, 0<t_{1}<t_{2}<1$, or $\omega(F \times(t, 1]), t<1$, or $\omega(F \times[0, t))$, $t>0$.

Suppose that $\beta=(E, B, p)$ is a fibre space. Let $\Sigma(E)$ be the quotient obtained from $E \times I$ by the identification

$$
(e, t) \sim\left(e^{\prime}, t\right) \text { if and only if } e=e^{\prime} \text { or } t=0 \text { or } 1 \text { and } p(e)=p\left(e^{\prime}\right) .
$$

Let $\omega: E \times I \rightarrow \Sigma(E)$ be the projection. A basis for the topology on $\Sigma(E)$ is to consist of sets of the form $\omega\left(U \times\left(t_{1}, t_{2}\right)\right), U$ open in $E, 0<t_{1}<t_{2}<1$, or $\omega\left(p^{-1}(V) \times\left(t_{1}, 1\right]\right), t<1$, or $\omega\left(p^{-1}(V) \times[0, t)\right), t>0$, where $V$ is open in $B$. Define

$$
\Sigma(p): \Sigma(E) \rightarrow B
$$

by $\Sigma(p)([e, t])=p(e)$.

(7.4) Lemma. If $\beta=(E, B, p)$ is locally trivial with fibre $F$, then $\Sigma(\beta)=$ $(\Sigma(E), B, \Sigma(p))$ is locally trivial with fibre $S(F)$.

This is easily checked. We will call $\Sigma(\beta)$ the suspension of $\beta$.

We will now describe a natural way of assigning to $\beta$ a $B$-spectrum. Let $\Sigma^{m}(\beta)$ $=\Sigma\left(\Sigma^{m-1}(\beta)\right)$ and define

$$
\Delta_{m}: B \rightarrow \Sigma^{m}(E)
$$

by $\Delta_{m}(b)=[e, 0 \cdots 0], e \in p^{-1}(b)$. Note that $\Delta_{m}$ is a cross-section to $\Sigma^{m}(p): \Sigma^{m}(E) \rightarrow B$.

Let $\mathscr{S}(\beta)$ denote the $B$-spectrum consisting of pairs $\left(\Gamma_{m}, \delta_{m}\right)$ and maps

$$
\left(k_{m}, K_{m}\right):\left(\Gamma_{m}, \delta_{m}\right) \rightarrow\left(\Omega\left(\Gamma_{m+1} ; \delta_{m+1}\right), \Omega\left(\delta_{m+1}\right)\right),
$$

where

$$
\begin{aligned}
\left(\Gamma_{m}, \delta_{m}\right) & =\left(\Sigma^{m}(\beta), \Delta_{m}\right), \quad m>0, \\
& =\left(\Omega^{-m+1}\left(\Sigma(\beta) ; \Delta_{1}\right), \Omega^{-m+1}\left(\Delta_{1}\right)\right), \quad m \leqq 0,
\end{aligned}
$$

and for $m>0$

$$
k_{m}: \Sigma^{m}(E) \rightarrow \Omega\left(\Sigma^{m+1}(E) ; \Delta_{m+1}\right)
$$


is defined by

and

$$
\begin{aligned}
k_{m}\left(\left[e, t_{1}, \ldots, t_{m}\right]\right)(\lambda) & =\left[\Delta_{m} p(e), 2 \lambda\right], \quad 0 \leqq \lambda \leqq 1 / 2, \\
& =\left[e, t_{1}, \ldots, t_{m}, 2-2 \lambda\right], \quad 1 / 2 \leqq \lambda \leqq 1,
\end{aligned}
$$

$$
K_{m}: B \times I \rightarrow \Omega\left(\Sigma^{m+1}(E), \Delta_{m+1}\right)
$$

is defined by

$$
\begin{aligned}
K_{m}(b, t)(\lambda) & =\left[\Delta_{m}(b), 2 t \lambda\right], \quad 0 \leqq \lambda \leqq 1 / 2, \\
& =\left[\Delta_{m}(b), 2 t(1-\lambda)\right], \quad 1 / 2 \leqq \lambda \leqq 1 ;
\end{aligned}
$$

whereas for $m \leqq 0$,

$$
k_{m}: \Omega^{-m+1}\left(\Sigma(E) ; \Delta_{1}\right) \rightarrow \Omega^{-m+1}\left(\Sigma(E) ; \Delta_{1}\right)
$$

is to be the identity, and

$$
K_{m}: B \times I \rightarrow \Omega^{-m+1}\left(\Sigma(E) ; \Delta_{1}\right)
$$

is to be the constant homotopy $K_{m}(b, t)=\Omega^{-m+1}\left(\Delta_{1}\right)(b), 0 \leqq t \leqq 1$.

The square of $\beta=(E, B, p)$ is $\beta^{2}=\left(E^{2}, E, p_{1}\right)$, where

$$
E^{2}=\left\{\left(e_{1}, e_{2}\right) \in E \times E \mid p\left(e_{1}\right)=p\left(e_{2}\right)\right\}
$$

and $p_{1}: E^{2} \rightarrow E$ is given by $p_{1}\left(e_{1}, e_{2}\right)=e_{1}$. There is a cross-section $\Delta: E \rightarrow E^{2}$ by $\Delta(e)=(e, e)$. Now define

$$
\mu: E^{2} \rightarrow \Omega\left(\Sigma(E) ; \Delta_{1}\right)
$$

by

In the diagrams

$$
\begin{aligned}
\mu\left(e_{1}, e_{2}\right)(\lambda) & =\left[e_{2}, 2 \lambda\right], \quad 0 \leqq \lambda \leqq 1 / 2, \\
& =\left[e_{1}, 2-2 \lambda\right], \quad 1 / 2 \leqq \lambda \leqq 1
\end{aligned}
$$

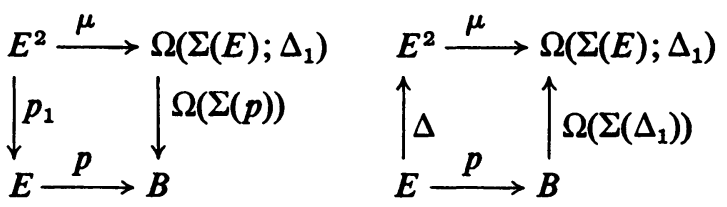

the first is commutative and the second is homotopy commutative, a connecting homotopy being

$$
M: E \times I \rightarrow \Omega\left(\Sigma(E) ; \Delta_{1}\right)
$$

by

$$
\begin{aligned}
M(e, t)(\lambda) & =[e, 2 t \lambda], \quad 0 \leqq \lambda \leqq 1 / 2, \\
& =[e, 2 t(1-\lambda)], \quad 1 / 2 \leqq \lambda \leqq 1 .
\end{aligned}
$$

Note that $M_{t}$ is a lifting of $p$ for $0 \leqq t \leqq 1$. 
Given $X \in \mathscr{P}^{2}$ and $f: X \rightarrow B$, let

$$
\mathscr{L}(X, f, \beta)=\{g: X \rightarrow E \mid p g=f\}
$$

and

$$
L(X, f, \beta)=\pi_{0}(\mathscr{L}(X, f, \beta)) .
$$

Suppose that $L(X, f, \beta)$ is not empty. Let $\alpha \in L(X, f, \beta)$ be represented by $g: X \rightarrow E$ and define

$$
\psi_{\alpha}: L(X, f, \beta) \rightarrow h^{\circ}(X, f, \mathscr{S}(\beta))
$$

to be the composition

$$
L(X, f, \beta) \stackrel{\eta(g)}{\longrightarrow} L\left(X, g, \beta^{2}\right) \stackrel{\mu_{\#}}{\longrightarrow} L\left(X, f, \Omega\left(\Sigma(\beta) ; \Delta_{1}\right)\right) \longrightarrow h^{0}(X, f, \mathscr{S}(\beta)),
$$

where $\eta(g)([q])=[g \times q], q \in \mathscr{L}(X, f, \beta)$, and the unmarked arrow is inclusion into the direct limit. Note that $\eta(g)$ is one-one and onto.

(7.20) LEMMA. The correspondence $\psi_{\alpha}$ is independent of the representative chosen for $\alpha$.

Proof. Let $g^{\prime}$ also represent $\alpha$ and let $H: X \times I \rightarrow E$ satisfy $H_{0}=g, H_{1}=g^{\prime}$ and $p H_{t}=f, 0 \leqq t \leqq 1$. Then, for $q \in \mathscr{L}(X, f, \beta)$, define

$$
J: X \times I \rightarrow \Omega\left(\Sigma(E) ; \Delta_{1}\right)
$$

by $J(x, t)=\mu(H(x, t), q(x)), x \in X, 0 \leqq t \leqq 1$. We have $J_{0}=\mu(g \times q), J_{1}=\mu\left(g^{\prime} \times q\right)$ and $\Omega(\Sigma(p)) J_{t}=f, 0 \leqq t \leqq 1$. Therefore $[\mu(g \times q)]=\left[\mu\left(g^{\prime} \times q\right)\right]$ in $L\left(X, f, \Omega\left(\Sigma(\beta) ; \Delta_{1}\right)\right)$. This completes the proof.

We need now the following fact. Suppose that we have a commutative diagram

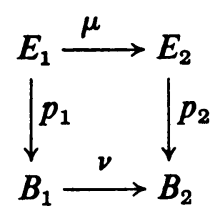

with both $p_{1}$ and $p_{2}$ fibre maps. Let $F_{1}$ and $F_{2}$ denote the respective fibres.

(7.22) Lemma. Suppose that

$$
\mu_{\#}: \pi_{m}\left(F_{1} ; e_{1}\right) \rightarrow \pi_{m}\left(F_{2} ; e_{2}\right)
$$

is an isomorphism, $m<2 n$. Then if $X \in \mathscr{P}^{2}$ is $(2 n-1)$-coconnected, the correspondence

$$
\mu_{\#}: L\left(X, f, \beta_{1}\right) \rightarrow L\left(X, v f, \beta_{2}\right), \quad f \in \mathscr{M}\left(X, B_{1}\right),
$$

is one-one and onto.

This is well known. 
(7.23) THEOREM. Let $\beta=(E, B, p)$ be locally trivial with fibre $F$. If $F$ is $(n-1)$ connected, $X \in \mathscr{P}^{2}$ is $(2 n-1)$-coconnected and $L(X, f, \beta)$ is not empty, then $\psi_{\alpha}$ in (7.18) is one-one and onto.

Proof. Apply the above lemma to conclude that both $\mu_{\#}$ in (7.19) and the inclusion of $L\left(X, f, \Omega\left(\Sigma(\beta) ; \Delta_{1}\right)\right)$ into the direct limit $h^{0}(X, f, \mathscr{S}(\beta))$ are one-one and onto.

With $X$ and $\beta$ as in the above theorem, let $(L(X, f, \beta), \alpha)$ denote the set $L(X, f, \beta)$ together with the abelian group structure determined by the condition that $\psi_{\alpha}$ be an isomorphism. For $\gamma_{1}, \gamma_{2} \in L(X, f, \beta)$, let $\gamma_{1}+_{\alpha} \gamma_{2}$ denote their sum in $(L(X, f, \beta), \alpha)$. Using the homotopy $M$ of (7.15), we see that $\alpha$ is the zero in this group.

(7.24) LemmA. For $\alpha_{0}, \alpha_{1} \in L(X, f, \beta)$ we have $\psi_{\alpha_{0}}\left(\alpha_{1}\right)=-\psi_{\alpha_{1}}\left(\alpha_{0}\right)$.

Proof. Let $g_{0}, g_{1}: X \rightarrow E$ represent $\alpha_{0}, \alpha_{1}$ respectively. Then $\psi_{\alpha_{0}}\left(\alpha_{1}\right)$ is represented by $\mu\left(g_{0} \times g_{1}\right)$ and $\psi_{\alpha_{1}}\left(\alpha_{0}\right)$ by $\mu\left(g_{1} \times g_{0}\right)$. From the definition of $\mu$, the product $\mu\left(g_{0} \times g_{1}\right) \cdot \mu\left(g_{1} \times g_{0}\right)$ is homotopic as a lifting of $f$ to the trivial lifting $\Omega\left(\Delta_{1}\right) f$. That is, $\psi_{\alpha_{0}}\left(\alpha_{1}\right)+\psi_{\alpha_{1}}\left(\alpha_{0}\right)=0$. This completes the proof.

(7.25) Lemma. For $\alpha_{0}, \alpha_{1} \in L(X, f, \beta)$ and $v \in h^{0}(X, f, \mathscr{S}(\beta))$, we have $\psi_{\alpha_{1}} \psi_{\alpha_{0}}^{-1}(v)$ $=\psi_{\alpha_{1}}\left(\alpha_{0}\right)+v$.

Proof. Let $q: X \rightarrow E$ be a lifting of $f$ such that $\mu\left(g_{0} \times q\right)$ represents $v$. Then $q$ represents $\psi_{\alpha_{0}}^{-1}(v)$ and $\psi_{\alpha_{1}} \psi_{\alpha_{0}}^{-1}(v)$ is represented by $\mu\left(g_{1} \times q\right)$. From the definition of $\mu$, we see that $\mu\left(g_{1} \times q\right)$ is homotopic as a lifting of $f$ to $\mu\left(g_{1} \times g_{0}\right) \cdot \mu\left(g_{0} \times q\right)$. The latter represents $\psi_{\alpha_{1}}\left(\alpha_{0}\right)+v$. This completes the proof.

(7.26) LemmA. For $\alpha_{0}, \alpha_{1}, \alpha_{2}, \gamma \in L(X, f, \beta)$, we have $\alpha_{0}+_{\alpha_{1}}\left(\alpha_{1}+\alpha_{2} \gamma\right)=\alpha_{0}+_{\alpha_{2}} \gamma$.

Proof. By (7.24) and (7.25), we have

Therefore

$$
\begin{aligned}
\psi_{\alpha_{1}}\left(\alpha_{1}+_{\alpha_{2}} \gamma\right) & =\psi_{\alpha_{1}} \psi_{\alpha_{2}}^{-1}\left(\psi_{\alpha_{2}}\left(\alpha_{1}\right)+\psi_{\alpha_{2}}(\gamma)\right) \\
& =\psi_{\alpha_{1}}\left(\alpha_{2}\right)+\psi_{\alpha_{2}}\left(\alpha_{1}\right)+\psi_{\alpha_{2}}(\gamma) \\
& =\psi_{\alpha_{2}}(\gamma) .
\end{aligned}
$$

$$
\begin{aligned}
\alpha_{0}+_{\alpha_{1}}\left(\alpha_{1}+_{\alpha_{2}} \gamma\right) & =\psi_{\alpha_{1}}^{-1}\left(\psi_{\alpha_{1}}\left(\alpha_{0}\right)+\psi_{\alpha_{1}}\left(\alpha_{1}+_{\alpha_{2}} \gamma\right)\right) \\
& =\psi_{\alpha_{1}}^{-1}\left(\psi_{\alpha_{1}}\left(\alpha_{0}\right)+\psi_{\alpha_{2}}(\gamma)\right) \\
& =\psi_{\alpha_{1}}^{-1}\left(\psi_{\alpha_{1}} \psi_{\alpha_{0}}^{-1} \psi_{\alpha_{2}}(\gamma)\right)=\psi_{\alpha_{0}}^{-1} \psi_{\alpha_{2}}(\gamma) .
\end{aligned}
$$

On the other hand, by (7.25)

$$
\begin{aligned}
\alpha_{0}+_{\alpha_{2}} \gamma & =\psi_{\alpha_{2}}^{-1}\left(\psi_{\alpha_{2}}\left(\alpha_{0}\right)+\psi_{\alpha_{2}}(\gamma)\right) \\
& =\psi_{\alpha_{2}}^{-1}\left(\psi_{\alpha_{2}} \psi_{\alpha_{0}}^{-1} \psi_{\alpha_{2}}(\gamma)\right)=\psi_{\alpha_{0}}^{-1} \psi_{\alpha_{2}}(\gamma) .
\end{aligned}
$$

Comparing (7.28) and (7.29) gives the desired result.

Let $\mathscr{U}$ denote the category of sets and functions, let $\mathscr{C}$ be an arbitrary category and let $F: \mathscr{C} \rightarrow \mathscr{U}$ be a contravariant functor. We say that $F$ has a natural affine group structure if for each object $X \in \mathscr{C}$ and element $\alpha \in F(X)$, there is a rule 
which assigns an abelian group structure to the set $F(X)$. Denote this group by $(F(X), \alpha)$ and for $\gamma_{1}, \gamma_{2} \in F(X)$ let $\gamma_{1}+{ }_{\alpha} \gamma_{2}$ denote their sum in $(F(X), \alpha)$. The following conditions must be satisfied.

(A). The zero of $(F(X), \alpha)$ is $\alpha$.

(B). If $g: X_{1} \rightarrow X_{2}$ is a map in $\mathscr{C}$, then $F(g):\left(F\left(X_{2}\right), \alpha\right) \rightarrow\left(F\left(X_{1}\right), F(g)(\alpha)\right)$ is a homomorphism.

(C). For $\alpha_{0}, \alpha_{1} \in F(X)$, the translation $T\left(\alpha_{0}, \alpha_{1}\right):\left(F(X), \alpha_{1}\right) \rightarrow\left(F(X), \alpha_{0}\right)$ defined by $T\left(\alpha_{0}, \alpha_{1}\right)(\gamma)=\alpha_{0}+\alpha_{1} \gamma$ is an isomorphism.

(D). $T\left(\alpha_{0}, \alpha_{0}\right)$ is the identity, $\alpha_{0} \in F(X)$.

(E). $T\left(\alpha_{0}, \alpha_{1}\right) T\left(\alpha_{1}, \alpha_{2}\right)=T\left(\alpha_{0}, \alpha_{2}\right), \alpha_{0}, \alpha_{1}, \alpha_{2} \in F(X)$.

Now let $\mathscr{P}(\beta, 2 n-1)$ denote the category whose objects are pairs $(X, f)$ with $X$ a $(2 n-1)$-coconnected CW-complex, $f \in \mathscr{M}(X, B)$ and $L(X, f, \beta)$ not empty. A map $g:\left(X_{1}, f_{1}\right) \rightarrow\left(X_{2}, f_{2}\right)$ in the category is to be $g: X_{1} \rightarrow X_{2}$ such that $f_{1}=f_{2} g$.

(7.30) THEOREM. Let $\beta=(E, B, p)$ be locally trivial with fibre $F$ which is $(n-1)$ connected. Then the set functor $L(X, f, \beta): \mathscr{P}(\beta, 2 n-1) \rightarrow \mathscr{U}$ has a natural affine group structure.

Proof. Properties (A) and (B) are easily checked. We will show now that

$$
T\left(\alpha_{0}, \alpha_{1}\right):\left(L(X, f, \beta), \alpha_{1}\right) \rightarrow\left(L(X, f, \beta), \alpha_{0}\right)
$$

is a homomorphism. By Lemma (7.26)

$$
\begin{aligned}
T\left(\alpha_{0}, \alpha_{1}\right)\left(\gamma_{2}+_{\alpha_{1}} \gamma_{2}\right) & =\alpha_{0}+{ }_{\alpha_{1}}\left(\gamma_{1}+_{\alpha_{1}} \gamma_{2}\right) \\
& =\left(\alpha_{0}+{ }_{\alpha_{1}} \gamma_{1}\right)+{ }_{\alpha_{0}}\left(\alpha_{0}+{ }_{\alpha_{1}} \gamma_{2}\right) \\
& =T\left(\alpha_{0}, \alpha_{1}\right)\left(\gamma_{1}\right)+{ }_{\alpha_{0}} T\left(\alpha_{0}, \alpha_{1}\right)\left(\gamma_{2}\right) .
\end{aligned}
$$

Property (D) is evident and (E) is just Lemma (7.26). Finally (D) and (E) imply that $T\left(\alpha_{0}, \alpha_{1}\right)$ is an isomorphism.

8. Equivariant maps. Let $G$ act as a group of transformations on $X$ and $Y$. A map $f: X \rightarrow Y$ is equivariant if $f(g x)=g f(x), g \in G, x \in X$. Two equivariant maps $f_{0}, f_{1}: X \rightarrow Y$ are equivariantly homotopic if there is a homotopy $F: X \times I \rightarrow Y$ from $f_{0}$ to $f_{1}$, such that $F_{t}$ is equivariant, $0 \leqq t \leqq 1$. Let $E(X, Y)$ denote the set of equivariant homotopy classes of equivariant maps from $X$ to $Y$. A map $f: X \rightarrow Y$ is an equivariant homotopy equivalence if there is $g: Y \rightarrow X$ such that $f g$ and $g f$ are equivariantly homotopic to the identity.

The following is an equivariant form of a theorem of J. H. C. Whitehead [10].

(8.1) Lemma. Suppose that $X$ and $Y$ are connected $C W$-complexes on which the action of $G$ is both free and cellular. If $f: X \rightarrow Y$ is equivariant and $f_{\#}: \pi_{m}\left(X ; x_{0}\right)$ $\rightarrow \pi_{m}\left(Y ; y_{0}\right)$ is an isomorphism, $m \leqq \max (\operatorname{dim} X, \operatorname{dim} Y)$, then $f$ is an equivariant homotopy equivalence.

The proof can be carried out, using the mapping cylinder of $f$, along the same lines as the proof of the Whitehead theorem. 
Let $W$ be a $G$-free acyclic complex. For any space $Z$ with an action of $G$, we have a locally trivial fibre space

$$
\beta_{z}=\left(W \times Z / G, W / G, \pi_{z}\right),
$$

where $G$ acts diagonally on $W \times Z$ and $\pi_{z}: W \times Z / G \rightarrow W / G$ is induced by projection. The fibre is $Z$.

(8.3) Lemma. Suppose $X$ is a $C W$-complex on which the action of $G$ is both free and cellular. Let $q: W \times X \rightarrow X$ be the projection. Then

$$
q^{\#}: E(W \times X, Y) \rightarrow E(X, Y)
$$

is one-one and onto.

Proof. This follows from Lemma (8.1), since $q_{\#}: \pi_{m}\left(W \times X,\left(w_{0}, x_{0}\right)\right) \rightarrow \pi_{m}\left(X ; x_{0}\right)$ is an isomorphism, $m \geqq 0$.

Let $X$ satisfy the hypothesis of the above lemma and let

$$
\varphi: E(X, Y) \rightarrow L\left(W \times X / G, \pi_{X}, \beta_{Y}\right)
$$

be the composition

$$
E(X, Y) \stackrel{q^{\#-1}}{\longrightarrow} E(W \times X, Y) \stackrel{\lambda}{\longrightarrow} L\left(W \times X / G, \pi_{X}, \beta_{Y}\right),
$$

where $\lambda$ is defined as follows. Let $\alpha \in E(W \times X, Y)$ be represented by $g: W \times X \rightarrow Y$. We have $\tilde{g}: W \times X \rightarrow W \times Y$ by $\tilde{g}(w, x)=(w, g(w, x))$ and $\tilde{g}$ is equivariant. Its orbit $\operatorname{map} \tilde{g} / G: W \times X / G \rightarrow W \times Y / G$ is a lifting of $\pi_{X}$. Let $\lambda(\alpha)$ be the class of $\tilde{g} / G$. The correspondence $\lambda$ is essentially due to A. Heller [3] and is one-one and onto. Therefore $\varphi$ is one-one and onto.

Fix a space $Y$ and an action of $G$ on $Y$. Let $\mathscr{Q}(Y, G, 2 n-1)$ denote the category whose objects are CW-complexes $X$ with an action of $G$ which is both free and cellular and such that $X / G$ is $(2 n-1)$-coconnected and $E(X, Y)$ is not empty. The maps in the category are to be equivariant maps. We then have a covariant functor

$$
D: \mathscr{Q}(Y, G, 2 n-1) \rightarrow \mathscr{P}\left(\beta_{Y}, 2 n-1\right)
$$

which sends $X$ to $\left(W \times X / G, \pi_{X}\right)$.

Suppose $Y$ is $(n-1)$-connected. There are the set functors $E(, Y)$ : $\mathscr{2}(Y, G, 2 n-1) \rightarrow \mathscr{U}$ and $L\left(, \beta_{Y}\right): \mathscr{P}\left(\beta_{Y}, 2 n-1\right) \rightarrow \mathscr{U}$ and $\varphi$ in (8.4) is a natural transformation $E(, Y) \rightarrow L\left(, \beta_{Y}\right) D$. Since $\varphi$ is one-one and onto, we may, for $X \in \mathcal{Q}(Y, G, 2 n-1)$ and $\alpha \in E(X, Y)$, define an abelian group $(E(X, Y), \alpha)$ with underlying set $E(X, Y)$ by the condition that

$$
\varphi:(E(X, Y), \alpha) \rightarrow\left(L\left(W \times X / G, \pi_{X}, \beta_{Y}\right), \varphi(\alpha)\right)
$$

be an isomorphism. Then from Theorem (7.30) we have

(8.7) THEOREM. Let $Y$ be an (n-1)-connected space with an action of the finite group $G$ on $Y$. Then the set functor $E(, Y): \mathscr{Q}(G, 2 n-1) \rightarrow \mathscr{U}$ has a natural affine group structure. 
REMARK. The addition in $(E(X, Y), \alpha)$ has a very simple description. Let $g, k_{1}, k_{2}: X \rightarrow Y$ represent $\alpha, \gamma_{1}, \gamma_{2}$ respectively. Let $G$ act diagonally on $Y^{3}$ and consider the equivariant map $g \times k_{1} \times k_{2}: X \rightarrow Y^{3}$. The subspace

$$
V(Y)=\left\{\left(y_{1}, y_{2}, y_{3}\right) \in Y^{3} \mid y_{1}=y_{2} \text { or } y_{1}=y_{3}\right\}
$$

is invariant and $\pi_{m}\left(Y^{3}, V(Y)\right)=0, m \leqq 2 n-1$. Since $X / G$ is $(2 n-1)$-coconnected we may construct a homotopy $H: X \times I \rightarrow Y^{3}$ such that $H_{0}=g \times k_{1} \times k_{2}, H_{1}(X)$ $\subset V(Y)$ and $H_{t}$ is equivariant, $0 \leqq t \leqq 1$. Define a folding map $\lambda: V(Y) \rightarrow Y$ by $\lambda\left(y, y_{2}, y\right)=y_{2}$ and $\lambda\left(y, y, y_{3}\right)=y_{3}$. A representative of $\gamma_{1}+\alpha \gamma_{2}$ is $\lambda H_{1}$. We will not need this fact so we will not stop to prove it.

Let $[X, Y]$ denote the track group of homotopy classes of maps from $X$ to $Y$ and let $\zeta: E(X, Y) \rightarrow[X, Y]$ assign to an equivariant homotopy class its ordinary homotopy class. Define

$$
\theta:(E(X, Y), \alpha) \rightarrow[X, Y]
$$

by $\theta(\gamma)=\zeta(\gamma)-\zeta(\alpha)$.

Fix base-points $w_{0} \in W$ and $y_{0} \in Y$. Let $i: X \rightarrow W \times X / G$ be given by $i(x)$ $=\left[w_{0}, x\right], x \in X$. This identifies $X$ with the fibre $\pi_{x}^{-1}\left(\left[w_{0}\right]\right)$. Let $w_{0}^{*}: X \rightarrow W / G$ and $\left(w_{0}, y_{0}\right)^{*}: X \rightarrow W \times Y / G$ be the constant maps at $\left[w_{0}\right]$ and $\left[w_{0}, y_{0}\right]$ respectively and let $z \in L\left(X, w_{0}^{*}, \beta_{Y}\right)$ be the class of $\left(w_{0}, y_{0}\right)^{*}$. Consider the diagram

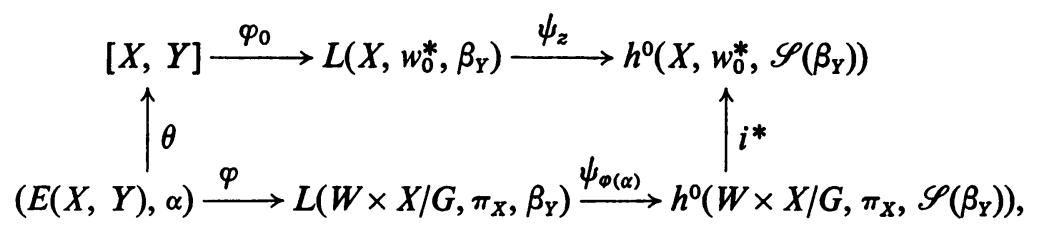

where $\varphi_{0}$ is defined as follows. Given $g: X \rightarrow Y$ define $g_{0}: X \rightarrow W \times Y / G$ by $g_{0}(x)$ $=\left[w_{0}, g(x)\right], x \in X$. Then let $\varphi_{0}(g)=\left[g_{0}\right]$.

We have an operation

$$
\rho: G \times[X, Y] \rightarrow[X, Y]
$$

of $G$ on $[X, Y]$ defined by $\rho(g, \gamma)=\left(g^{-1}\right)^{\#} g_{\#}(\gamma), g \in G, \gamma \in[X, Y]$.

Next, we have a fibre map $\pi_{X}: W \times X / G \rightarrow W / G$ with fibre $X$. Take $f \in$ $\mathscr{M}(W / G, W / G)$ to be the identity. Then from $\S 4$, the collection $h^{0}\left(\pi_{\bar{X}}^{-1}([w]), \pi_{X}, \mathscr{S}\left(\beta_{Y}\right)\right)$, $[w] \in W / G$, is a local system over $W / G$. Out of this we obtain an operation

$$
\tilde{\rho}: \pi_{1}\left(W / G ;\left[w_{0}\right]\right) \times h^{0}\left(X, w_{0}^{*}, \mathscr{S}\left(\beta_{Y}\right)\right) \rightarrow h^{0}\left(X, w_{0}^{*}, \mathscr{S}\left(\beta_{Y}\right)\right) .
$$

Make the canonical identification of $G$ with $\pi_{1}\left(W / G ;\left[w_{0}\right]\right)$.

(8.12) LemMa. The diagram (8.9) is commutative and

$$
\psi_{z} \varphi_{0}:[X, Y] \rightarrow h^{0}\left(X, w_{0}^{*}, \mathscr{S}\left(\beta_{Y}\right)\right)
$$

is an operator isomorphism. 
The proof is tedious but straightforward and will be omitted. As a consequence of the lemma, $\theta$ is a homomorphism.

For a group $A$ with $G$ as left operators, let $I(A)$ denote its subgroup of invariant elements. Note that the image of $\theta$ is contained in $I([X, Y])$. For an integer $n$, let $\mathscr{A}(n)$ denote the class of abelian torsion groups whose $p$-primary component is zero if $p$ does not divide $n$.

(8.13) THEOREM. Let $G$ have order $n$. Then

$$
\theta:(E(X, Y), \alpha) \rightarrow I([X, Y])
$$

is an isomorphism modulo $\mathscr{A}(n)$.

Proof. By the preceding lemma it is sufficient to show that

$$
i^{*}: h^{0}\left(W \times X / G, \pi_{X}, \mathscr{S}\left(\beta_{Y}\right)\right) \rightarrow I\left(h^{0}\left(X, w_{0}^{*}, \mathscr{S}\left(\beta_{Y}\right)\right)\right)
$$

is an isomorphism modulo $\mathscr{A}(n)$. Applying the spectral sequence of $\S 4$ to $\pi_{X}$ : $W \times X / G \rightarrow W / G$, we have

$$
E_{2}^{p, q}=H^{p}\left(W / G ;\left[h^{q}\left(X, \mathscr{S}\left(\beta_{Y}\right)\right)\right]\right)
$$

and a finite filtration

$$
h^{0}\left(W \times X / G, \pi_{X}, \mathscr{S}\left(\beta_{Y}\right)\right)=J^{0,0} \supset J^{1,-1} \supset \ldots \supset J^{k,-k} \supset \ldots
$$

with $E_{\infty}^{k,-k}=E_{r}^{k,-k}$ for large $r$.

We need the well-known facts [6] that $H^{p}\left(W / G ;\left[h^{q}\left(X, \mathscr{S}\left(\beta_{Y}\right)\right)\right]\right)$ is in $\mathscr{A}(n), p>0$, and

$$
H^{\circ}\left(W / G ;\left[h^{q}\left(X, \mathscr{S}\left(\beta_{Y}\right)\right)\right]\right) \simeq I\left(h^{q}\left(X, w_{0}^{*}, \mathscr{S}\left(\beta_{Y}\right)\right)\right) .
$$

From the above filtration we have that $i^{*}$ in (8.14) is an isomorphism modulo $\mathscr{A}(n)$. This completes the proof.

Suppose $G=Z_{2}$ and $Y=S^{n}$, where the action of $Z_{2}$ on $S^{n}$ is given by the antipodal map. Let $\Sigma^{n}(X)$ denote the $n$th stable cohomotopy group of $X$.

(8.15) Corollary. Let $T$ be a cellular fixed point free involution on the $C W$ complex $X$ with $X / T(2 n-1)$-coconnected. Then

$$
\theta:\left(E\left(X, S^{n}\right), \alpha\right) \rightarrow I\left(\Sigma^{n}(X)\right)
$$

is an isomorphism modulo 2-torsion.

Let $\omega: \Sigma^{n}(X) \rightarrow H^{n}(X)$ be the Hopf map and let $Q$ denote the rational numbers. A theorem of Serre [8] asserts that

$$
\omega \otimes 1: \Sigma^{n}(X) \otimes Q \rightarrow H^{n}(X ; Q)
$$

is an isomorphism.

Let $Z_{2}$ operate on $H^{n}(X ; Q)$ by the rule $U \rightarrow T^{*}(u), n$-odd, and $U \rightarrow-T^{*}(u)$, n-even. 
(8.17) Corollary. With $X$ and $T$ as in (8.15)

$$
\omega \theta \otimes 1:\left(E\left(X, S^{n}\right), \alpha\right) \otimes Q \rightarrow I\left(H^{n}(X ; Q)\right)
$$

is an isomorphism.

Proof. Note that with the operation defined above on $H^{n}(X ; Q), \omega \otimes 1$ is an operator isomorphism. Now apply (8.15).

9. Immersions and embeddings. For a closed $C^{\infty}$-manifold $M$ of dimension $n$ let $T(M)$ and $T_{0}(M)$ denote respectively its tangent bundle and tangent sphere bundle. Let $E^{n+k}$ denote Euclidean $(n+k)$-space. An immersion $f: M \rightarrow E^{n+k}$ is a $C^{\infty}$-map whose derivative $T(f): T(M) \rightarrow T\left(E^{n+k}\right)$ has rank $n$ at each point $x \in M$. Two immersions $f_{0}, f_{1}: M \rightarrow E^{n+k}$ are regularly homotopic if there is a $C^{\infty}$-map $F: M \times I \rightarrow E^{n+k}$ such that $F_{0}=f_{0}, F_{1}=f_{1}$, and $F_{t}$ is an immersion, $0 \leqq t \leqq 1$. Let $I M^{n+k}(M)$ denote the set of regular homotopy classes of immersions of $M$ into $E^{n+k}$.

An embedding $f: M \rightarrow E^{n+k}$ is a one-one immersion. Two embeddings $f_{0}, f_{1}: M$ $\rightarrow E^{n+k}$ are isotopic if there is a $C^{\infty}$-map $F: M \times I \rightarrow E^{n+k}$ such that $F_{0}=f_{0}$, $F_{1}=f_{1}$ and $F_{t}$ is an embedding, $0 \leqq t \leqq 1$. Let $E M^{n+k}(M)$ denote the set of isotopy classes of embeddings of $M$ into $E^{n+k}$.

There is a fixed point free involution $A_{M}$ on $T_{0}(M)$ which on each fibre $S^{n-1}$ is the antipodal map $A_{m-1}$.

Let $\Delta$ denote the diagonal of $M \times M$. There is a fixed point free involution $B_{M}$ on $M \times M-\Delta$ defined by $(x, y) \rightarrow(y, x)$.

An immersion $f: M \rightarrow E^{n+k}$ determines an equivariant map $T_{0}(f): T_{0}(M)$ $\rightarrow E^{n+k} \times S^{n+k-1}$. Since the projection $\pi: E^{n+k} \times S^{n+k-1} \rightarrow S^{n+k-1}$ is equivariant, so also is $\pi T_{0}(f): T_{0}(M) \rightarrow S^{n+k-1}$.

An embedding $f: M \rightarrow E^{n+k}$ gives an equivariant map $f \times f: M \times M-\Delta \rightarrow E^{n+k}$ $\times E^{n+k}-\Delta$. There is $\lambda: E^{n+k} \times E^{n+k}-\Delta \rightarrow S^{n+k-1}$ by $\lambda\left(v_{1}, v_{2}\right)=v_{1}-v_{2} /\left|v_{1}-v_{2}\right|$ and $\lambda$ is equivariant. Then $\lambda(f \times f): M \times M-\Delta \rightarrow S^{n+k-1}$ is also equivariant.

Our study of the sets $I M^{n+k}(M)$ and $E M^{n+k}(M)$ is based on the following

(9.1) ThEOREM (HIRSCH-HAEFLIGER [5]). Suppose $2 k>n+1$. The correspondence

$$
\eta: I M^{n+k}(M) \rightarrow E\left(T_{0}(M) ; S^{n+k-1}\right)
$$

defined by $\eta([f])=\left[\pi T_{0}(f)\right]$ is one-one and onto.

(9.2) THEOREM (HAEFLIGER [4]). Suppose $2 k>n+3$. The correspondence

$$
\tau: E M^{n+k}(M) \rightarrow E\left(M \times M-\Delta ; S^{n+k-1}\right)
$$

defined by $\tau([f])=[\lambda(f \times f)]$ is one-one and onto.

By means of $\eta$ and $\tau$ the sets $I M^{n+k}(M)$ and $E M^{n+k}(M)$ inherit a natural affine group structure. 
(9.3) TheOREM. For $k>1$,

$$
A_{M}^{\#}: \Sigma^{n+k-1}\left(T_{0}(M)\right) \rightarrow \Sigma^{n+k-1}\left(T_{0}(M)\right)
$$

is $(-1)^{n}$ times the identity, modulo 2-torsion.

Proof. There is a spectral sequence $\left\{E_{r}\right\}$ with

and a filtration

$$
E_{2}^{p, q}=H^{p}\left(M ;\left[\Sigma^{q}\left(S^{n-1}\right)\right]\right)
$$

$$
\Sigma^{n+k-1}\left(T_{0}(M)\right)=J^{0, n+k-1} \supset \ldots \supset J^{n, k-1} \supset 0
$$

with $J^{p, q} / J^{p+1, q-1}=E_{\infty}^{p, q}$. It is sufficient to show that for $q>0$, the induced automorphism of $E_{2}^{p, q}$ is $(-1)^{n}$ times the identity. This agrees with the coefficient automorphism determined by $A_{m-1}^{\#}: \Sigma^{q}\left(S^{n-1}\right) \rightarrow \Sigma^{q}\left(S^{n-1}\right)$, since $A_{m-1}$ is the restriction of $A_{M}$ to the fibre. It is well known that for $q>0, A_{m-1}^{\#}$ is $(-1)^{n}$ times the identity. This completes the proof.

Letting $I\left(\Sigma^{n+k-1}\left(T_{0}(M)\right)\right)$ denote the subgroup of elements invariant under $\left(A_{m+k-1}\right)_{\#} A_{M}^{\#}$, we have by the above lemma

(9.4) CorollaRY. Let $k>1$. For $k$ even, $I\left(\Sigma^{n+k-1}\left(T_{0}(M)\right)\right)=\Sigma^{n+k-1}\left(T_{0}(M)\right)$ and for $k$ odd, $I\left(\Sigma^{n+k-1}\left(T_{0}(M)\right)\right)=0$, modulo 2-torsion.

We will write $M \subseteq E^{n+k}\left(M \subset E^{n+k}\right)$ if there exists an immersion (embedding) of $M$ in $E^{n+k}$. Applying (8.15) and the preceding corollary, we have

(9.5) Theorem. Suppose $2 k>n+1$ and $M \subseteq E^{n+k}$. For $k$-odd $\left(\operatorname{IM}^{n+k}(M), \alpha\right)=0$ modulo 2-torsion. For $k$-even

$$
\theta_{\eta}:\left(I M^{n+k}(M), \alpha\right) \rightarrow \Sigma^{n+k-1}\left(T_{0}(M)\right)
$$

is an isomorphism modulo 2-torsion.

For embeddings we have

(9.6) TheOREM. Suppose $2 k>n+3$ and $M \subset E^{n+k}$. Then

$$
\theta \tau:\left(E M^{n+k}(M), \alpha\right) \rightarrow I\left(\Sigma^{n+k-1}(M \times M-\Delta)\right)
$$

is an isomorphism modulo 2-torsion.

Here $I\left(\Sigma^{n+k-1}(M \times M-\Delta)\right)$ is the subgroup of elements invariant under $\left(A_{m+k-1}\right)_{\#} B_{M}^{\#}$.

10. Rank of $I M^{n+k}(M)$ and $E M^{n+k}(M)$. In this section it is assumed that $M$ is orientable. Let

$$
\tilde{\omega}:\left(I M^{n+k}(M), \alpha\right) \rightarrow H^{k}(M)
$$

be the composition

$$
\left(I M^{n+k}(M), \alpha\right) \stackrel{\theta \eta}{\longrightarrow} \Sigma^{n+k-1}\left(T_{0}(M)\right) \stackrel{\omega}{\longrightarrow} H^{n+k-1}\left(T_{0}(M)\right) \stackrel{\psi}{\longrightarrow} H^{k}(M)
$$

where $\psi$ is from the Gysin sequence for $T_{0}(M) \rightarrow M$. 
For an immersion $g: M \rightarrow E^{n+k}$, the normal class of $g$ is the Euler class $\chi(g) \in H^{k}(M)$ of the normal bundle of $g$.

(10.2) LEMMA. Let $\gamma$ generate $H^{n+k-1}\left(S^{n+k-1}\right)$. Then with a suitable orientation of the normal bundle of $g$, we have

$$
\chi(g)=\psi T_{0}(g)^{*} \pi^{*}(\gamma) .
$$

Proof. This follows from Theorem (1.1) of [7]. Let $g^{-1}\left(T_{0}\left(E^{n+k}\right)\right)$ be the bundle over $M$ induced by $g$ and let

$$
T_{0}(M) \stackrel{f_{2}}{\longrightarrow} g^{-1}\left(T_{0}\left(E^{n+k}\right)\right) \stackrel{\tilde{g}}{\longrightarrow} T_{0}\left(E^{n+k}\right)
$$

be the factorization of $T_{0}(g)$. Then

$$
\psi T_{0}(g)^{*} \pi^{*}(\gamma)=\psi f_{2}^{*} \tilde{g}^{*} \pi^{*}(\gamma)
$$

In the Gysin sequence for $g^{-1}\left(T_{0}\left(E^{n+k}\right)\right) \rightarrow M$, we have $\psi \tilde{g}^{*} \pi^{*}(\gamma)=1 \in H^{0}(M)$. Using the notation of Theorem (1.1) of [7], we have

$$
\psi f_{2}^{*} \tilde{g}^{*} \pi^{*}(\gamma)=G_{2} \psi \tilde{g}^{*} \pi^{*}(\gamma)=G_{2}^{*}(1)=\chi(g) .
$$

This completes the proof.

The above lemma implies that $\chi(g)$ depends only on the class $\beta \in I M^{n+k}(M)$, so we will write $\chi(\beta)$ instead of $\chi(g)$.

(10.4) LEMMA. For $\gamma \in I M^{n+k}(M), \tilde{\omega}(\gamma)=\chi(\gamma)-\chi(\alpha)$.

Proof. This follows from the preceding lemma and the definition of $\tilde{\omega}$.

Now from Theorem (9.5) we have

(10.5) THEOREM. Suppose $2 k>n+1$ and $M \subseteq E^{n+k}$. For $k$-odd, $\left(I^{n+k}(M), \alpha\right)$ $\otimes Q=0$ and for $k$-even

$$
\tilde{\omega} \otimes 1:\left(I M^{n+k}(M), \alpha\right) \otimes Q \rightarrow H^{k}(M ; Q),
$$

given by $\tilde{\omega} \otimes 1(\gamma \otimes x)=(\chi(\gamma)-\chi(\alpha)) \otimes x$, is an isomorphism.

For embeddings, let

$$
\tilde{\omega}:\left(E M^{n+k}(M), \alpha\right) \rightarrow H^{n+k-1}(M \times M-\Delta)
$$

be the composition

$$
\left(E M^{n+k}(M), \alpha\right) \stackrel{\theta \tau}{\longrightarrow} \Sigma^{n+k-1}(M \times M-\Delta) \stackrel{\omega}{\longrightarrow} H^{n+k-1}(M \times M-\Delta) .
$$

By Theorem (8.15) we have an isomorphism

$\tilde{\omega} \otimes 1:\left(E M^{n+k}(M), \alpha\right) \otimes Q \rightarrow I\left(H^{n+k-1}(M \times M-\Delta ; Q)\right)$. 
Let $u \in H_{2 n}(M \times M)$ be a fundamental class and let

$$
D: H^{n+k-1}(M \times M-\Delta ; Q) \rightarrow H_{n-k+1}(M \times M, \Delta ; Q)
$$

denote the Lefschetz-Poincaré duality map

$$
D(v)=u \cap v, \quad v \in H^{n+k-1}(M \times M-\Delta ; Q) .
$$

Next, let

$$
\kappa: H_{*}(M ; Q) \otimes H_{*}(M ; Q) \rightarrow H_{*}(M \times M ; Q)
$$

be the Künneth map and $\delta: M \rightarrow M \times M$ the diagonal map. For $a \in H_{n-k+1}(M)$, write

$$
\begin{aligned}
& \kappa^{-1} \delta_{*}(a)=a \otimes 1 \oplus 1 \otimes a+\hat{a}, \\
& \hat{a} \in \sum_{i=1}^{n-k} H_{i}(M ; Q) \otimes H_{i^{\prime}}(M ; Q),
\end{aligned}
$$

$i^{\prime}=n-k+1-i$. The element $a$ is primitive if $\hat{a}=0$. Let $j: M \times M \rightarrow(M \times M, \Delta)$ be the inclusion. We have an isomorphism

$$
\left(j_{*} \kappa\right)^{-1} D: H^{n+k-1}(M \times M-\Delta ; Q) \rightarrow \sum_{i=1}^{n-k+1} H_{i}(M ; Q) \otimes H_{i^{\prime}}(M ; Q)
$$

Define an involution $T$ on the right hand side of $(10.10)$ by

$$
\begin{array}{ll}
T(a \otimes 1)=(-a) \otimes 1-\hat{a}, & \operatorname{dim}(a)=n-k+1, \\
T(a \otimes b)=(-1)^{i i^{\prime}} b \otimes a, & \operatorname{dim}(a)=i, \operatorname{dim}(b)=i^{\prime}>0 .
\end{array}
$$

Then $\left(j_{*} \kappa\right)^{-1} D$ is an operator isomorphism when the involution on the left is $(-1)^{n+k} B_{M}^{*}$ and on the right is $(-1)^{k} T$. Now let

$$
J_{n, k}(M ; Q)=\sum_{i=1}^{r} H_{i}(M ; Q) \otimes H_{i^{\prime}}(M ; Q),
$$

where $r$ is the greatest integer less than or equal to $n-k / 2$ and let $P_{n-k+1}(M ; Q)$ denote the subgroup of primitive elements in $H_{n-k+1}(M ; Q)$.

(10.13) THEOREM Suppose $2 k>n+3$ and $M \subset E^{n+k}$. Then $\left(E M^{n+k}(M), \alpha\right) \otimes Q$ is given by the following table:

\begin{tabular}{|l|l|l|}
\cline { 2 - 3 } \multicolumn{1}{c|}{} & $k \equiv 0 \bmod 2$ & $k \equiv 1 \bmod 2$ \\
\hline$n+k \equiv 0,1,2 \bmod 4$ & $J_{n, k}(M ; Q)$ & $J_{n, k}(M ; Q) \oplus P_{n-k+1}(M ; Q)$ \\
\hline$n+k \equiv 3 \bmod 4$ & $J_{n, k}(M ; Q) \oplus H_{n-k+1 / 2}(M ; Q)$ & $J_{n, k}(M ; Q) \oplus H_{n-k+1 / 2}(M ; Q)$ \\
& & $\oplus P_{n-k+1}(M ; Q)$ \\
\hline
\end{tabular}


Proof. The various cases are all handled in the same way. For example, when $n+k \equiv 0,1,2 \bmod 4$ and $k \equiv 0 \bmod 2$,

by

$$
\rho: J_{n, k}(M ; Q) \rightarrow \sum_{i=1}^{n-k+1} H_{i}(M ; Q) \otimes H_{i^{\prime}}(M ; Q)
$$

$$
\rho\left(\sum_{i=1}^{r} a_{i} \otimes b_{i^{\prime}}\right)=\sum_{i=1}^{r}\left(a_{i} \otimes b_{i^{\prime}} \oplus(-1)^{i i^{\prime}} b_{i^{\prime}} \otimes a_{i}\right)
$$

is injective onto the subgroup of elements invariant under $(-1)^{k} T$.

11. The normal class of an immersion. In this section, $M$ is orientable. We consider a question raised by Lashof and Smale [7] as to what elements $v \in H^{k}(M)$ are realizable as normal classes of an immersion. Such elements will be characterized as permanent cycles in a spectral sequence.

If $M \subseteq E^{n+k}$ let

$$
N^{k}(M)=\left\{v \in H^{k}(m) \mid v=\chi(\gamma), \gamma \in I M^{n+k}(M)\right\} .
$$

If $2 k>n+1$, then by Lemma (10.4), $N^{k}(M)$ is a coset of $\tilde{\omega}\left(I M^{n+k}(M), \alpha\right)$. If it is assumed that $M \subseteq E^{n+k-1}$ or $M \subset E^{n+k}$, then there is $\alpha \in I M^{n+k}(M)$ such that $\chi(\alpha)=0$. In this case $N^{k}(M)=\tilde{\omega}\left(I M^{n+k}(M), \alpha\right)$ and is therefore a subgroup of $H^{k}(M)$.

Let $S^{\infty}$ and $P^{\infty}$ be the infinite dimensional sphere and projective space respectively, let $X(M)=S^{\infty} \times T_{0}(M) / Z_{2}$ and $P_{0}(M)=T_{0}(M) / Z_{2}$ and let $\pi_{1}: X(M)$ $\rightarrow P^{\infty}$ and $\pi_{2}: X(M) \rightarrow P_{0}(M)$ be the projections. Pick $s_{0} \in S^{\infty}$ and define $i: T_{0}(M)$ $\rightarrow X(M)$ by $i(x)=\left[s_{0}, x\right], x \in T_{0}(M)$. From the definition of $\tilde{\omega}$ and the commutativity of (8.9) we see that the image of $\tilde{\omega}$ is equal to the image of the composition

$$
\begin{aligned}
& h^{0}\left(X(M), \pi_{1}, \mathscr{S}(\beta)\right) \stackrel{i^{*}}{\longrightarrow} \Sigma^{n+k-1}\left(T_{0}(M)\right) \\
& \stackrel{\omega}{\longrightarrow} H^{n+k-1}\left(T_{0}(M)\right) \stackrel{\psi}{\longrightarrow} H^{k}(M),
\end{aligned}
$$

where $\beta=\left(S^{\infty} \times S^{n+k-1} / Z_{2}, P^{\infty}, \pi_{1}\right)$.

Constructing the spectral sequence for the identity map $X(M) \rightarrow X(M)$ and $\pi_{1} \in \mathscr{M}\left(X(M), P^{\infty}\right)$, we have

(11.3) $E_{2}^{p, q}=H^{p}\left(X(M) ;\left[\Sigma^{q+n+k-1}(p t)\right]\right)=H^{p}\left(P_{0}(M) ;\left[\Sigma^{q+n+k-1}(p t)\right]\right)$

(the right-hand identification being made by $\pi_{2}^{*}$ ) and

(11.4) $j: h^{0}\left(X(M), \pi_{1}, \mathscr{S}(\beta)\right) \rightarrow E_{\infty}^{n+k-1,-(n+k-1)} \subset H^{n+k-1}\left(P_{0}(M) ;[Z]\right)$.

Then the following diagram is commutative.

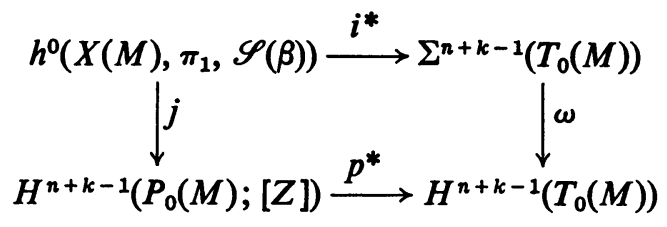


where $p: T_{0}(M) \rightarrow P_{0}(M)$ is the orbit map. Combining the above facts, we have

(11.6) THEOREM. Suppose $2 k>n+1$ and $M \subset E^{n+k}$ or $M \subseteq E^{n+k-1}$. Then

$$
N^{k}(M)=\psi p^{*}\left(E_{\infty}^{n+k-1,-(n+k-1)}\right) .
$$

(11.7) Lemma. For $k$-odd $\psi p^{*}\left(H^{n+k-1}\left(P_{0}(M) ;[Z]\right)\right)=0 . \quad$ For $k$-even, $\psi p^{*}\left(H^{n+k-1}\left(P_{0}(M) ;[Z]\right)\right) \subset 2 H^{k}(M)$.

Proof. Comparing the spectral sequence for $T_{0}(M) \rightarrow M$ and $P_{0}(M) \rightarrow M$, we have a commutative diagram

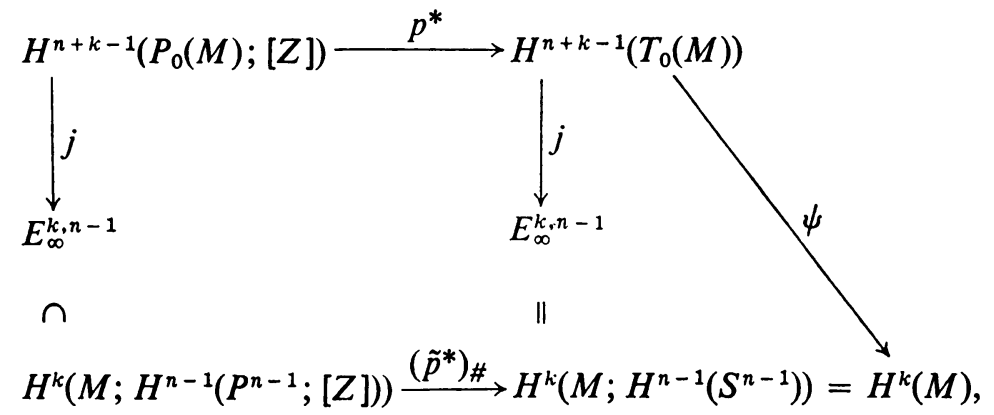

where $\tilde{p}: S^{n-1} \rightarrow P^{n-1}$ is the restriction of $p$ to the fibre. The involution on $Z$ is $(-1)^{n+k}$. Thus $H^{n-1}\left(P^{n-1} ;[Z]\right)$ is $Z_{2}$ or $Z$ depending on whether $k$ is odd or even. In the former case $\tilde{p}^{*}$ has image 0 and in the latter $\tilde{p}^{*}$ has image $2 H^{n-1}\left(S^{n-1}\right)$. The lemma now follows from the commutativity of the above diagram.

From (11.6), (11.7) and Theorem (10.6), we have

(11.8) TheOREM. Suppose $2 k>n+1$ and $M \subseteq E^{n+k-1}$. For $k$-odd, $N^{k}(M)=0$. For $k$-even, $N^{k}(M)$ is a subgroup of $2 H^{k}(M)$ having finite index.

REMARK. For $k=n$ or $n-1$ and $k$-even, we deduce that

$$
\psi p^{*}\left(H^{n+k-1}\left(P_{0}(M) ;[Z]\right)\right)=2 H^{k}(M) .
$$

Then using Theorem.(11.6), we obtain the following table for $N^{k}(M)$ :

\begin{tabular}{|l|c|c|}
\cline { 2 - 3 } \multicolumn{1}{c|}{} & $n$-even & $n$-odd \\
\hline$k=n$ & $2 H^{n}(M)$ & 0 \\
\hline$k=n-1$ & 0 & $2 H^{n-1}(M)$ \\
\hline
\end{tabular}

The values for $k=n$ were given by Lashof and Smale [7]. Information for $k<n-1$ would involve computing the twisted cohomology operations which appear as differential operators in the spectral sequence.

Let $S^{k} \times S^{n-1}$ have the involution $(a, b) \rightarrow(a,-b)$.

(11.9) LEMMA. For k-even, there is an equivariant map $f: S^{k} \times S^{n-1} \rightarrow S^{k+n-1}$ of degree 2. 
Proof. Let $p: S^{k} \times S^{n-1} \rightarrow S^{k} \times P^{n-1}$ be the orbit map. There is an equivariant map $g: S^{k} \times S^{n-1} \rightarrow S^{k+n-1}$ of degree 0 , namely, the projection onto $S^{n-1}$ followed by the inclusion $S^{n-1} \subset S^{k+n-1}$. Now the correspondence

$$
E\left(\bar{S}^{k} \times S^{n-1}, S^{k+n-1}\right) \rightarrow H^{k+n-1}\left(S^{k} \times P^{n-1} ;[Z]\right)
$$

which assigns to $f$ the primary obstruction $d(f, g)$ to an equivariant homotopy between $f$ and $g$ is one-one and onto. The involution on $Z$ is $(-1)^{k+n}=(-1)^{n}$ and, by an elementary computation, $H^{k+n-1}\left(S^{k} \times P^{n-1} ;[Z]\right)=Z$ and

$$
p^{*}: H^{k+n-1}\left(S^{k} \times P^{n-1} ;[Z]\right) \rightarrow H^{k+n-1}\left(S^{k} \times S^{n-1}\right)
$$

takes a generator to twice a generator. Therefore if we choose $f$ so that $d(f, g)$ is a generator, the degree of $f$ will be 2 .

Suppose that $M$ is parallelizable. Then $T_{0}(M)=M \times S^{n-1}$ and we can define

$$
\psi: \Sigma^{n+k-1}\left(T_{0}(M)\right) \rightarrow \Sigma^{k}(M)
$$

to be the composition

$$
\Sigma^{n+k-1}\left(M \times S^{n-1}\right) \stackrel{j^{*-1}}{\longrightarrow} \Sigma^{n+k-1}\left(M \times S^{n-1}, M \times S^{n-1}\right) \longrightarrow \Sigma^{k}(M),
$$

where $j$ is inclusion and the unmarked arrow is $(n-1)$-fold desuspension. The following diagram is commutative:

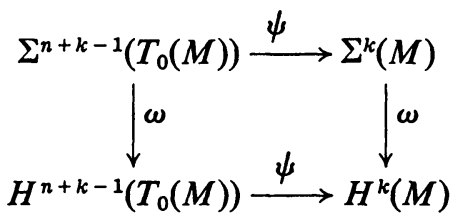

(11.12) THEOREM. Suppose $M$ is parallelizable and $2 k>n+1$. Then

$$
\psi \theta \eta:\left(I M^{n+k}(M), \alpha\right) \rightarrow \Sigma^{k}(M)
$$

has image $0, k$-odd, and $2 \Sigma^{k}(M), k$-even.

Proof. We will first show that the image of

$$
i^{*}: h^{0}\left(X(M), \pi_{1}, \mathscr{S}(\beta)\right) \rightarrow \Sigma^{n+k-1}\left(T_{0}(M)\right)
$$

is $0, k$-odd, and is contained in $2 \Sigma^{n+k-1}\left(T_{0}(M)\right), k$-even. This will imply that the image of $\psi \theta \eta$ is $0, k$-odd, and contained in $2 \Sigma^{k}(N)$, $k$-even. We have a commutative diagram

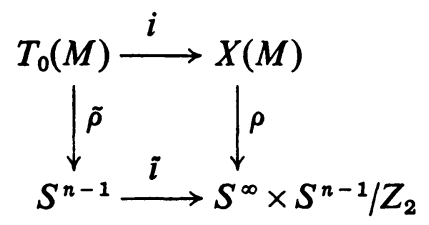


where $\rho$ and $\tilde{\rho}$ are projections and $\tilde{l}$ is inclusion. Comparing the spectral sequences for $\rho$ and $\tilde{\rho}$, we obtain a commutative diagram

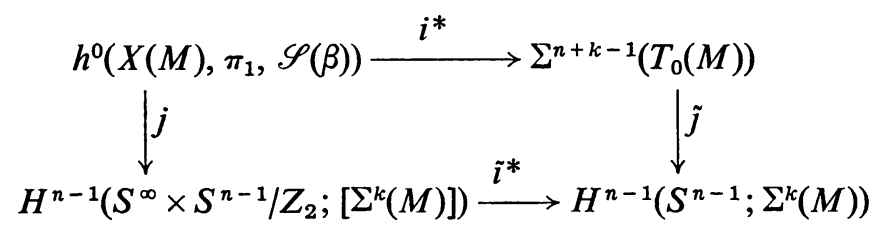

Since $\tilde{j}$ is an isomorphism and $i^{*}$ has image $0, k$-odd, and $2 H^{n-1}\left(S^{n-1} ; \Sigma^{k}(M)\right)$, $k$-even, it follows that the image of $i^{*}$ is $0, k$-odd, and contained in $2 \Sigma^{n+k-1}\left(T_{0}(M)\right)$, $k$-even.

Suppose now that $k$ is even. Let $u \in 2 \Sigma^{k}(M)$ and choose $f: M \rightarrow S^{k}$ such that $u=2[f]$. Let $f^{\prime}: M \times S^{n-1} \rightarrow S^{k} \times S^{n-1}$ be defined by $f^{\prime}(x, b)=(f(x), b)$. Then $f^{\prime}$ is equivariant when the involution on $M \times S^{n-1}$ is $(x, b) \rightarrow(x,-b)$ and on $S^{k} \times S^{n-1}$ is $(a, b) \rightarrow(a,-b)$. By Lemma (11.9), there is an equivariant map $g: S^{k} \times S^{n-1} \rightarrow S^{n+k-1}$ of degree 2. Let $\gamma \in E\left(T_{0}(M), S^{n+k-1}\right)$ be the class of $g f^{\prime}$. Then choosing $\alpha \in I M^{n+k}(M)$ so that $\zeta \eta(\alpha)=0$ in $\Sigma^{n+k-1}\left(T_{0}(M)\right.$ ), (see (8.8)), we have

$$
\psi \theta \eta\left(\eta^{-1}(\gamma)\right)=2[f]=u .
$$

Therefore, when $k$ is even, the image of $\psi \theta \eta$ is onto $2 \Sigma^{k}(M)$. This completes the proof.

Let $S^{k}(M)$ denote the subgroup of spherical classes of $H^{k}(M)$, that is, the image of $\omega: \Sigma^{k}(M) \rightarrow H^{k}(M)$.

(11.13) Corollary. Suppose $M$ is parallelizable and $2 k>n+1$. Then $N^{k}(M)=0$, $k$-odd, and $N^{k}(M)=2 S^{k}(M), k$-even.

Proof. Choose $\alpha \in I M^{n+k}(M)$ such that $\zeta \eta(\alpha)=0$. Suppose $v \in N^{k}(M)$. Let $\gamma \in I M^{n+k}(M)$ be such that $v=\chi(\gamma)$. Then by Lemma $(10.4), v=\tilde{\omega}(\gamma)$. By the preceding theorem, there is $u \in \Sigma^{k}(M)$ such that $\psi \theta \eta(\gamma)=2 u$. Then, by the commutativity of (11.11),

$$
v=\tilde{\omega}(\gamma)=2 \omega(u) \in 2 S^{k}(M) .
$$

Conversely, suppose $v \in 2 S^{k}(M)$. Let $u \in \Sigma^{k}(M)$ be such that $v=2 \omega(u)$. By the preceding theorem there is $\gamma \in I M^{n+k}(M)$ such that $\psi \theta \eta(\gamma)=2 u$. By the commutativity of (9.12)

$$
\chi(\gamma)=\tilde{\omega}(\gamma)=\omega(2 u)=v .
$$

This completes the proof.

REMARK. Theorems (11.12) and (11.13) are also true if $M$ is a $\pi$-manifold. The proofs are essentially the same.

\section{BIBLIOGRAPHY}

1. W. Barcus, Note on cross-sections over $C W$-complexes, Quart. J. Math. Oxford Ser. (2) 5 (1954), 150-160. 
2. A. Dold, Relations between ordinary and extraordinary cohomology theories, Colloquium on algebraic topology, Aarhus Universitet, 1962, pp. 2-9.

3. A. Heller, On equivariant maps of spaces with operators, Ann. of Math. 55 (1952), 223-231.

4. A. Haefliger, Plongements différentiables dans le domaine stable, Comment. Math. Helv. 37 (1962), 155-176.

5. A. Haefliger and M. Hirsch, Immersions in the stable range, Ann. of Math. 75 (1962), 231-241.

6. P. Hilton and S. Wylie, Homology theory, Cambridge Univ. Press, New York, 1960.

7. R. Lashof and S. Smale, On the immersion of manifolds in euclidean space, Ann. of Math. 68 (1958), 562-583.

8. J.-P. Serre, Groupes d'homotopie et classes de groupes abéliens, Ann. of Math. 58 (1953), 258-294.

9. G. W. Whitehead, Generalized homology theories, Trans. Amer. Math. Soc. 102 (1962), 227-283.

10. J. H. C. Whitehead, Combinatorial homotopy. I, Bull. Amer. Math. Soc. 55 (1949), 213-245.

UNIVERSITY OF MASSACHUSETTS,

AMHERST, MASSACHUSETTS 